\title{
Higher Hölder regularity for nonlocal equations with irregular kernel
}

\author{
Simon Nowak ${ }^{1}$
}

Received: 5 July 2020 / Accepted: 28 December 2020 / Published online: 18 January 2021

(c) The Author(s) 2021

\begin{abstract}
We study the higher Hölder regularity of local weak solutions to a class of nonlinear nonlocal elliptic equations with kernels that satisfy a mild continuity assumption. An interesting feature of our main result is that the obtained regularity is better than one might expect when considering corresponding results for local elliptic equations in divergence form with continuous coefficients. Therefore, in some sense our result can be considered to be of purely nonlocal type, following the trend of various such purely nonlocal phenomena observed in recent years. Our approach can be summarized as follows. First, we use certain test functions that involve discrete fractional derivatives in order to obtain higher Hölder regularity for homogeneous equations driven by a locally translation invariant kernel, while the global behaviour of the kernel is allowed to be more general. This enables us to deduce the desired regularity in the general case by an approximation argument.
\end{abstract}

Mathematics Subject Classification 35R09 · 35B65 · 35D30 · 47G20

\section{Introduction}

\subsection{Basic setting and main result}

In this work, we study the higher Hölder regularity of solutions to nonlinear nonlocal equations of the form

$$
L_{A}^{\Phi} u=f \text { in } \Omega \subset \mathbb{R}^{n}
$$

driven by a kernel that potentially exhibits a very irregular behaviour. More precisely, by modifying an approach introduced in [2], we prove that so-called local weak solutions to such equations are locally Hölder continuous with some explicitly determined Hölder exponent.

Communicated by A. Malchiodi.

Supported by SFB 1283 of the German Research Foundation.

Simon Nowak

simon.nowak@uni-bielefeld.de

1 Fakultät für Mathematik, Universität Bielefeld, Postfach 100131, 33501 Bielefeld, Germany 
Here $s \in(0,1), \Omega \subset \mathbb{R}^{n}$ is a domain (= open set), $f: \mathbb{R}^{n} \rightarrow \mathbb{R}$ is a given function and

$$
L_{A}^{\Phi} u(x):=2 \lim _{\varepsilon \rightarrow 0} \int_{\mathbb{R}^{n} \backslash B_{\varepsilon}(x)} \frac{A(x, y)}{|x-y|^{n+2 s}} \Phi(u(x)-u(y)) d y, \quad x \in \Omega,
$$

is a nonlocal operator. Throughout the paper, for simplicity we assume that $n>2 s$. Furthermore, the function $A: \mathbb{R}^{n} \times \mathbb{R}^{n} \rightarrow \mathbb{R}$ is measurable and we assume that there exists a constant $\lambda \geq 1$ such that

$$
\lambda^{-1} \leq A(x, y) \leq \lambda \text { for almost all } x, y \in \mathbb{R}^{n} .
$$

Moreover, we require $A$ to be symmetric, i.e.

$$
A(x, y)=A(y, x) \text { for almost all } x, y \in \mathbb{R}^{n} .
$$

We call such a function $A$ a kernel coefficient. We define $\mathcal{L}_{0}(\lambda)$ as the class of all such measurable kernel coefficients $A$ that satisfy the conditions (2) and (3). Moreover, in our main results $\Phi: \mathbb{R} \rightarrow \mathbb{R}$ is assumed to be a continuous function satisfying $\Phi(0)=0$ and the following Lipschitz continuity and monotonicity assumptions, namely

$$
\left|\Phi(t)-\Phi\left(t^{\prime}\right)\right| \leq \lambda\left|t-t^{\prime}\right| \text { for all } t, t^{\prime} \in \mathbb{R}
$$

and

$$
\left(\Phi(t)-\Phi\left(t^{\prime}\right)\right)\left(t-t^{\prime}\right) \geq \lambda^{-1}\left(t-t^{\prime}\right)^{2} \text { for all } t, t^{\prime} \in \mathbb{R},
$$

where for simplicity we use the same constant $\lambda \geq 1$ as in (2). In particular, if $\Phi(t)=t$, then the operator $L_{A}^{\Phi}$ reduces to a linear nonlocal operator which is widely considered in the literature. The above conditions are for example satisfied by any $C^{1}$ function $\Phi$ with $\Phi(0)=0$ such that the image of the first derivative $\Phi^{\prime}$ of $\Phi$ is contained in $\left[\lambda^{-1}, \lambda\right]$.

Define the fractional Sobolev space

$$
W^{s, 2}(\Omega):=\left\{u \in L^{2}(\Omega) \mid \int_{\Omega} \int_{\Omega} \frac{|u(x)-u(y)|^{2}}{|x-y|^{n+2 s}} d y<\infty\right\}
$$

and denote by $W_{l o c}^{s, 2}(\Omega)$ the set of all functions $u \in L_{l o c}^{2}(\Omega)$ that belong to $W^{s, 2}\left(\Omega^{\prime}\right)$ for any relatively compact open subset $\Omega^{\prime}$ of $\Omega$. In addition, we define the tail space

$$
L_{2 s}^{1}\left(\mathbb{R}^{n}\right):=\left\{u \in L_{l o c}^{1}\left(\mathbb{R}^{n}\right) \mid \int_{\mathbb{R}^{n}} \frac{|u(y)|}{1+|y|^{n+2 s}} d y<\infty\right\} .
$$

We remark that for any function $u \in L_{2 s}^{1}\left(\mathbb{R}^{n}\right)$, the quantity

$$
\int_{\mathbb{R}^{n} \backslash B_{R}\left(x_{0}\right)} \frac{|u(y)|}{\left|x_{0}-y\right|^{n+2 s}} d y
$$

is finite for all $R>0, x_{0} \in \mathbb{R}^{n}$. For all measurable functions $u, \varphi: \mathbb{R}^{n} \rightarrow \mathbb{R}$, we define

$$
\mathcal{E}_{A}^{\Phi}(u, \varphi):=\int_{\mathbb{R}^{n}} \int_{\mathbb{R}^{n}} \frac{A(x, y)}{|x-y|^{n+2 s}} \Phi(u(x)-u(y))(\varphi(x)-\varphi(y)) d y d x,
$$

provided that the above expression is well-defined and finite. This is for example the case if $u \in W_{l o c}^{s, 2}(\Omega) \cap L_{2 s}^{1}\left(\mathbb{R}^{n}\right)$ and $\varphi \in W_{c}^{s, 2}(\Omega)$, where by $W_{c}^{s, 2}(\Omega)$ we denote the set of all functions that belong to $W^{s, 2}(\Omega)$ and are compactly supported in $\Omega$.

In the literature, various types of weak solutions with varying generality are considered. In this paper, we adopt the following very general notion of local weak solutions which is for example used in [1] and [2]. 
Definition Let $f \in L_{l o c}^{\frac{2 n}{n+2 s}}(\Omega)$. We say that $u \in W_{l o c}^{s, 2}(\Omega) \cap L_{2 s}^{1}\left(\mathbb{R}^{n}\right)$ is a local weak solution of the equation $L_{A}^{\Phi} u=f$ in $\Omega$, if

$$
\mathcal{E}_{A}^{\Phi}(u, \varphi)=(f, \varphi)_{L^{2}(\Omega)} \quad \forall \varphi \in W_{c}^{s, 2}(\Omega) .
$$

We remark that the right-hand side of (6) is finite by the fractional Sobolev embedding (cf. [9, Theorem 6.5]). It is noteworthy that the above notion of local weak solutions contains most other notions of weak solutions considered in the literature, such as the ones considered in e.g. [8] or [22].

In our first main result, we are going to impose an additional continuity assumption on $A$. Namely, we assume that there exists some small $\varepsilon>0$ such that

$$
\lim _{h \rightarrow 0} \sup _{\substack{x, y \in K \\|x-y| \leq \varepsilon}}|A(x+h, y+h)-A(x, y)|=0 \text { for any compact set } K \subset \Omega .
$$

In particular, the condition (7) is satisfied if $A$ is either continuous close to the diagonal in $\Omega \times \Omega$ or if $A$ belongs to the following subclass of $\mathcal{L}_{0}(\lambda)$ which plays an important role in our proof of the desired regularity.

Definition Let $\Omega$ be a domain and $\lambda \geq 1$. We say that a kernel coefficient $A_{0} \in \mathcal{L}_{0}(\lambda)$ belongs to the class $\mathcal{L}_{1}(\lambda, \Omega)$, if there exists a measurable function $a: \mathbb{R}^{n} \rightarrow \mathbb{R}$ such that $A_{0}(x, y)=a(x-y)$ for all $x, y \in \Omega$.

A kernel coefficient that belongs to the class $\mathcal{L}_{1}(\lambda, \Omega)$ can be thought of being translation invariant, but only inside of $\Omega$. We also call such a kernel coefficient locally translation invariant. We note that the condition (7) is also satisfied by some more general choices of kernel coefficients, for example if

$$
A(x, y)=A^{\prime}(x, y) A_{0}(x, y),
$$

where $A^{\prime} \in \mathcal{L}_{0}\left(\lambda^{\frac{1}{2}}\right)$ is continuous near the diagonal in $\Omega \times \Omega$ and $A_{0}$ belongs to the class $\mathcal{L}_{1}\left(\lambda^{\frac{1}{2}}, \Omega\right)$, but is not required to satisfy any continuity or smoothness assumption. Moreover, we stress that the condition given by (7) only restricts the behaviour of $A$ close to the diagonal in $\Omega \times \Omega$, while away from the diagonal in $\Omega \times \Omega$ and outside of $\Omega \times \Omega$ a more general behaviour is possible.

We are now in the position to state our main results.

Theorem 1.1 Let $\Omega \subset \mathbb{R}^{n}$ be a domain, $s \in(0,1), \lambda \geq 1$ and $f \in L_{\text {loc }}^{q}(\Omega)$ for some $q>\frac{n}{2 s}$. Consider a kernel coefficient $A \in \mathcal{L}_{0}(\lambda)$ that satisfies the condition (7) for some $\varepsilon>0$ and suppose that $\Phi$ satisfies (4) and (5) with respect to $\lambda$. Moreover, assume that $u \in W_{\text {loc }}^{s, 2}(\Omega) \cap L_{2 s}^{1}\left(\mathbb{R}^{n}\right)$ is a local weak solution of the equation $L_{A}^{\Phi} u=f$ in $\Omega$. Then for any $0<\alpha<\min \left\{2 s-\frac{n}{q}, 1\right\}$, we have $u \in C_{l o c}^{\alpha}(\Omega)$.

Furthermore, for all $R>0, x_{0} \in \Omega$ such that $B_{R}\left(x_{0}\right) \Subset \Omega$ and any $\sigma \in(0,1)$, we have

$$
\begin{aligned}
{[u]_{C^{\alpha}\left(B_{\sigma R}\left(x_{0}\right)\right) \leq} \leq \frac{C}{R^{\alpha}} } & \left(R^{-\frac{n}{2}}\|u\|_{L^{2}\left(B_{R}\left(x_{0}\right)\right)}+R^{2 s} \int_{\mathbb{R}^{n} \backslash B_{R}\left(x_{0}\right)} \frac{|u(y)|}{\left|x_{0}-y\right|^{n+2 s}} d y\right. \\
& \left.+R^{2 s-\frac{n}{q}}\|f\|_{L^{q}\left(B_{R}\left(x_{0}\right)\right)}\right),
\end{aligned}
$$

where $C=C(n, s, \lambda, \alpha, q, \sigma, \varepsilon)>0$ and

$$
[u]_{C^{\alpha}\left(B_{\sigma R}\left(x_{0}\right)\right)}:=\sup _{\substack{x, y \in B_{\sigma R}\left(x_{0}\right) \\ x \neq y}} \frac{|u(x)-u(y)|}{|x-y|^{\alpha}} .
$$


If we focus on obtaining Hölder regularity for some fixed exponent $0<\alpha<\min \left\{2 s-\frac{n}{q}, 1\right\}$, then we can slightly weaken the assumption on $A$ as follows. Roughly speaking, in this case it is enough to require that $A$ is locally close enough to being translation invariant, while the condition (7) essentially means that $A$ is locally arbitrarily close to being translation invariant. This slight "room for error" is typical when one uses approximation techniques in order to obtain regularity results, see for example [5].

Theorem 1.2 Let $\Omega \subset \mathbb{R}^{n}$ be a domain, $s \in(0,1), \lambda \geq 1$ and $f \in L_{\text {loc }}^{q}(\Omega)$ for some $q>\frac{n}{2 s}$. Consider a kernel coefficient $A \in \mathcal{L}_{0}(\lambda)$ and suppose that $\Phi$ satisfies (4) and (5) with respect to $\lambda$. Fix some $0<\alpha<\min \left\{2 s-\frac{n}{q}, 1\right\}$. Then there exists some small enough $\delta=\delta(\alpha, n, s, \lambda, q)>0$, such that if for any $z \in \Omega$, there exists some small enough radius $r_{z}>0$ and some $A_{z} \in \mathcal{L}^{1}\left(\lambda, B_{r_{z}}(z)\right)$ such that

$$
\left\|A-A_{z}\right\|_{L^{\infty}\left(B_{r_{z}}(z) \times B_{r_{z}}(z)\right)} \leq \delta,
$$

then for any local weak solution $u \in W_{\text {loc }}^{s, 2}(\Omega) \cap L_{2 s}^{1}\left(\mathbb{R}^{n}\right)$ of the equation $L_{A}^{\Phi} u=f$ in $\Omega$, we have $u \in C_{\text {loc }}^{\alpha}(\Omega)$. Moreover, for all $R>0, x_{0} \in \Omega$ such that $B_{R}\left(x_{0}\right) \Subset \Omega$ and any $\sigma \in(0,1), u$ satisfies the estimate (8) with respect to $\alpha$ and some constant $C=C\left(n, s, \lambda, \alpha, q, \sigma,\left\{r_{z}\right\}_{z \in \Omega}\right)>0$.

Remark 1.3 In order to provide some context, let us briefly consider the local elliptic equation in divergence form of the type

$$
\operatorname{div}(B \nabla u)=0 \text { in } \Omega,
$$

where the matrix of coefficients $B=\left\{b_{i j}\right\}_{i, j=1}^{n}$ is assumed to be uniformly elliptic and bounded. The equation (9) can in some sense be thought of as a local analogue of the nonlocal equation (1) corresponding to the limit case $s=1$. A classical regularity result states that if the coefficients $b_{i j}$ are continuous, then weak solutions $u \in W_{l o c}^{1,2}(\Omega)$ of the equation (9) are locally Hölder continuous for any exponent $\alpha \in(0,1)$, see for example [13, Corollary 5.18]. Heuristically, one might therefore expect that the optimal regularity in the setting of nonlocal equations with continuous kernel coefficient should not exceed $C^{s}$ regularity. Nevertheless, Theorem 1.1 in particular shows that weak solutions to nonlocal equations of the type $L_{A}^{\Phi} u=0$ in $\Omega$ are locally $C^{\alpha}$ for any $0<\alpha<\min \{2 s, 1\}$ whenever $A \in \mathcal{L}_{0}(\lambda)$ is continuous, exceeding $C^{s}$ regularity. In particular, in the case when $s \geq 1 / 2$, weak solutions to homogeneous nonlocal equations with continuous kernel coefficients enjoy the same amount of Hölder regularity as weak solutions to corresponding local equations with continuous coefficients, despite the fact that the order of such nonlocal equations is lower.

Such at first sight unexpected additional regularity is however not untypical in the context of nonlocal equations and has been observed in various previous works in the context of Sobolev regularity. For example, in [18] and [24] it is shown that already in the setting of a general kernel coefficient $A \in \mathcal{L}_{0}(\lambda)$, weak solutions to nonlocal equations of the type (1) are slightly higher differentiable than initially assumed along the scale of Sobolev spaces, which is a phenomenon not shared by local elliptic equations of the type (9) with coefficients that are merely measurable.

Another result in this direction was recently proved in [21], where the authors in particular show that if $A \in \mathcal{L}_{0}(\lambda)$ is Hölder continuous with some arbitrary Hölder exponent and $\Phi(t)=t$, then weak solutions of the equation $L_{A}^{\Phi} u=0$ in $\mathbb{R}^{n}$ belong to $W_{\text {loc }}^{\alpha, p}\left(\mathbb{R}^{n}\right)$ for any $\alpha<\min \{2 s, 1\}$ and any $2 \leq p<\infty$, while for local equations of the type (9) with corresponding Hölder continuous coefficients no comparable gain in differentiability is achievable. In particular, by the Sobolev embedding this result implies that such weak 
solutions belong to $C_{l o c}^{\alpha}\left(\mathbb{R}^{n}\right)$ for any $0<\alpha<\min \{2 s, 1\}$, which is consistent with our main result. Our main result shows that this amount of higher Hölder regularity is also enjoyed by local weak solutions of possibly nonlinear equations driven by kernel coefficients of class $\mathcal{L}_{0}(\lambda)$ that satisfy the continuity assumption (7).

Remark 1.4 Besides being interesting for its own sake, one of our main motivations is that Theorem 1.1 also has some interesting potential applications concerning the Sobolev regularity of solutions to nonlocal equations. A first such application can briefly be summarized as follows. In [22], in the main result it is assumed that $A$ is globally translation invariant, i.e. that $A$ belongs to the class $\mathcal{L}_{1}\left(\lambda, \mathbb{R}^{n}\right)$. However, this assumption is only used in order to ensure that the Hölder estimate (8) from Theorem 1.1 is valid, which up to this point was only known for translation invariant kernels, cf. [22, Theorem 4.6]. Since otherwise the proofs in [22] only rely on the properties (2) and (3) of $A$, from Theorem 1.1 above we conclude that the statement of [22, Theorem 1.1] is also true for general kernel coefficients $A$ of class $\mathcal{L}_{0}(\lambda)$ that satisfy the condition (7).

\subsection{Approach and previous results}

As mentioned, our approach is strongly influenced by an approach introduced in [2], where a similar result concerning higher Hölder regularity is proved for the fractional $p$-Laplacian in the superquadratic case when $p \geq 2$. Although for simplicity we restrict ourselves to the quadratic case when $p=2$, in contrast to [2] we deal with a nonlinearity already in the quadratic setting and most importantly, we also treat equations driven by general kernel coefficients $A$ that satisfy the mild assumption (7), while in [2] only the case when $A \equiv 1$ is considered. Also, we stress that by combining our techniques with some more techniques from [2], our approach could be modified in order to treat also nonlinearities with nonlinear growth of the type $\Phi(t) \approx t^{p-1}$. However, since the additional difficulties arising from such a generalization were already dealt with in [2] and we instead want to focus on the difficulties arising from considering equations with general coefficients, we decided not to pursue this direction in this work.

Let us briefly summarize our approach, highlighting the differences to the one used in [2]. First, we prove the higher Hölder regularity for homogeneous equations driven by a locally translation invariant kernel coefficient, see Sect. 3. As in [2], the main idea in this case is to test the equation with certain monotone power functions of discrete fractional derivatives leading to an incremental higher integrability and differentiability result on the scale of certain Besov-type spaces. However, in our setting we also need to carefully use the local translation invariance and the bounds imposed on $A$, and also the assumptions (4) and (5) imposed on $\Phi$ in order to overcome the difficulties that arise due to the presence of the general kernel and the general type of nonlinearity. Moreover, we remark that restricting ourselves to equations with linear growth has the advantage that the proof of this incremental higher regularity result simplifies quite substantially in some other respects. The obtained incremental gain in regularity is then iterated, in order for the desired Hölder regularity to follow by embedding.

In Sect. 4, we then treat the general case of inhomogeneous equations driven by a kernel coefficient satisfying the condition (7) by an approximation argument. In the corresponding approximation argument applied in [2], the solution is approximated by a solution of a corresponding equation with zero right-hand side, while the nonlocal operator driving the equation is left unchanged. In order to be able to treat equations with a general kernel coefficient $A$ of class $\mathcal{L}_{0}(\lambda)$ that satisfies only the continuity assumption (7), in addition to freezing the right-hand side, we also need to locally replace $A$ by a corresponding locally translation 
invariant kernel coefficient, which is possible in view of the assumption (7). Since by the first part of the proof the desired Hölder regularity is already known for solutions to equations with locally translation invariant kernel coefficients, we can then transfer this regularity from the approximate solution to the solution itself. In other words, in some sense we locally freeze the coefficient, in order to transfer the regularity from an equation for which the higher regularity can be proved directly to an equation driven by a less regular kernel. This strategy can be thought of as a nonlocal counterpart of corresponding techniques widely used in the study of higher regularity for local elliptic equations, although we stress that in our nonlocal setting we have to overcome a number of additional difficulties which are not present in the local setting in order to execute such an approximation argument successfully. Moreover, we believe that just like in the local setting, the approximation techniques developed in this paper are flexible enough in order to be adaptable to also proving other higher regularity results for nonlocal equations similar to (1).

Regarding other related regularity results, in [12] a similar result is proved in the linear case when $\Phi(t)=t$, where $A$ is required to be locally close enough to $b\left(\frac{x-y}{|x-y|}\right)$ for some even function $b: S^{n-1} \rightarrow \mathbb{R}$ that is bounded between two positive constants, which is contained in our assumption on $A$ in Theorem 1.2. More results concerning higher Hölder regularity for various types of nonlocal equations are for instance contained in $[5,6,11,23]$ and [14]. Furthermore, results regarding basic Hölder regularity for nonlocal equations are proved for example in $[8,15,19,25]$, while results concerning Sobolev regularity can be found for example in $[1,7,10,18,21,22,24]$. Finally, for some regularity results concerning nonlocal equations similar to (1) in the more general setting of measure data, we refer to [17].

\section{Preliminaries}

\subsection{Some notation}

Let us fix some notation which we use throughout the paper. By $C, c, C_{i}$ and $c_{i}, i \in \mathbb{N}_{0}$, we always denote positive constants, while dependences on parameters of the constants will be shown in parentheses. As usual, by

$$
B_{r}\left(x_{0}\right):=\left\{x \in \mathbb{R}^{n}|| x-x_{0} \mid<r\right\}, \quad \bar{B}_{r}\left(x_{0}\right):=\left\{x \in \mathbb{R}^{n}|| x-x_{0} \mid \leq r\right\}
$$

we denote the open and closed ball with center $x_{0} \in \mathbb{R}^{n}$ and radius $r>0$, respectively. Moreover, if $E \subset \mathbb{R}^{n}$ is measurable, then by $|E|$ we denote the $n$-dimensional Lebesguemeasure of $E$. If $0<|E|<\infty$, then for any $u \in L^{1}(E)$ we define

$$
\bar{u}_{E}:=f_{E} u(x) d x:=\frac{1}{|E|} \int_{E} u(x) d x .
$$

Next, for any $p \in(1, \infty)$ we define the function $J_{p}: \mathbb{R} \rightarrow \mathbb{R}$ by

$$
J_{p}(t):=|t|^{p-2} t
$$

Moreover, for any measurable function $\psi: \mathbb{R}^{n} \rightarrow \mathbb{R}$ and any $h \in \mathbb{R}^{n}$, we define 


$$
\begin{aligned}
\psi_{h}(x) & :=\psi(x+h), \quad \delta_{h} \psi(x):=\psi_{h}(x)-\psi(x), \\
\delta_{h}^{2}(x) & :=\delta_{h}\left(\delta_{h} \psi(x)\right)=\psi_{2 h}(x)+\psi(x)-2 \psi_{h}(x) .
\end{aligned}
$$

\subsection{The nonlocal tail}

In this section, for convenience we state and proof the following two simple results concerning the nonlocal tail of a function which we use frequently throughout the paper.

Lemma 2.1 Let $s \in(0,1)$ and $0<r<R$. Then for any $x \in \bar{B}_{r}$ and any $u \in L_{2 s}^{1}\left(\mathbb{R}^{n}\right)$, we have

$$
\int_{\mathbb{R}^{n} \backslash B_{R}} \frac{|u(y)|}{|x-y|^{n+2 s}} d y \leq\left(\frac{R}{R-r}\right)^{n+2 s} \int_{\mathbb{R}^{n} \backslash B_{R}} \frac{|u(y)|}{|y|^{n+2 s}} d y .
$$

Proof The claim follows directly from the observation that for any $x \in \bar{B}_{r}$ and any $y \in$ $\mathbb{R}^{n} \backslash B_{R}$, we have

$$
|y| \leq|x-y|+|x|=|x-y|\left(1+\frac{|x|}{|x-y|}\right) \leq|x-y|\left(1+\frac{r}{R-r}\right)=\frac{R}{R-r}|x-y| .
$$

Lemma 2.2 Let $s \in(0,1), r>0$ and $x_{0} \in B_{1}$ such that $B_{r}\left(x_{0}\right) \subset B_{1}$. Then for any $u \in L_{2 s}^{1}\left(\mathbb{R}^{n}\right)$, we have

$$
\int_{\mathbb{R}^{n} \backslash B_{r}\left(x_{0}\right)} \frac{|u(y)|}{\left|x_{0}-y\right|^{n+2 s}} d y \leq r^{-(n+2 s)}\left(\|u\|_{L^{1}\left(B_{1}\right)}+\int_{\mathbb{R}^{n} \backslash B_{1}} \frac{|u(y)|}{|y|^{n+2 s}} d y\right) .
$$

Proof Since by assumption $x_{0} \in \bar{B}_{1-r}$, with the help of Lemma 2.1 we obtain

$$
\begin{aligned}
\int_{\mathbb{R}^{n} \backslash B_{r}\left(x_{0}\right)} \frac{|u(y)|}{\left|x_{0}-y\right|^{n+2 s}} d y & =\int_{B_{1} \backslash B_{r}\left(x_{0}\right)} \frac{|u(y)|}{\left|x_{0}-y\right|^{n+2 s}} d y+\int_{\mathbb{R}^{n} \backslash B_{1}} \frac{|u(y)|}{\left|x_{0}-y\right|^{n+2 s}} d y \\
& \leq r^{-(n+2 s)}|| u \|_{L^{1}\left(B_{1}\right)}+r^{-(n+2 s)} \int_{\mathbb{R}^{n} \backslash B_{1}} \frac{|u(y)|}{|y|^{n+2 s}} d y,
\end{aligned}
$$

which finishes the proof.

\subsection{The fractional Sobolev space $W^{s, 2}$}

First of all, for notational convenience for any domain $\Omega \subset \mathbb{R}^{n}$ we define the seminorm associated to the space $W^{s, 2}(\Omega)$ by

$$
[u]_{W^{s, 2}(\Omega)}:=\left(\int_{\Omega} \int_{\Omega} \frac{|u(x)-u(y)|^{2}}{|x-y|^{n+2 s}} d y d x\right)^{1 / 2},
$$

so that we have

$$
W^{s, 2}(\Omega)=\left\{u \in L^{2}(\Omega) \mid[u]_{W^{s, 2}(\Omega)}<\infty\right\} .
$$

Moreover, we define the space

$$
W_{0}^{s, 2}(\Omega):=\left\{u \in W^{s, 2}\left(\mathbb{R}^{n}\right) \mid u \equiv 0 \text { in } \mathbb{R}^{n} \backslash \Omega\right\} .
$$

The following Poincaré-type inequality associated to the space $W^{s, 2}$ will frequently be used throughout the paper. 
Lemma 2.3 (fractional Friedrichs-Poincaré inequality) Let $s \in(0,1)$ and consider a bounded domain $\Omega \subset \mathbb{R}^{n}$. For any $u \in W_{0}^{s, 2}(\Omega)$, we have

$$
\int_{\Omega}|u(x)|^{2} d x \leq C|\Omega|^{\frac{2 s}{n}} \int_{\mathbb{R}^{n}} \int_{\mathbb{R}^{n}} \frac{|u(x)-u(y)|^{2}}{|x-y|^{n+2 s}} d y d x,
$$

where $C=C(n, s)>0$.

Proof Since $u \in W_{0}^{s, 2}(\Omega) \subset W^{s, 2}\left(\mathbb{R}^{n}\right)$ and $n>2 s$, applying Hölder's inequality and then the fractional Sobolev inequality (cf. [9, Theorem 6.5]) leads to

$$
\begin{aligned}
\int_{\Omega}|u(x)|^{2} d x & \leq|\Omega|^{\frac{2 s}{n}}\left(\int_{\Omega}|u(x)|^{\frac{2 n}{n-2 s}} d x\right)^{\frac{n-2 s}{n}} \\
& \leq C|\Omega|^{\frac{2 s}{n}} \int_{\mathbb{R}^{n}} \int_{\mathbb{R}^{n}} \frac{|u(x)-u(y)|^{2}}{|x-y|^{n+2 s}} d y d x,
\end{aligned}
$$

where $C=C(n, s)>0$. This finishes the proof.

\subsection{Besov-type spaces}

Next, let us introduce some function spaces of Besov-type. In order to do so, for $q \in[1, \infty)$ and any function $u \in L^{q}\left(\mathbb{R}^{n}\right)$ we define the quantities

$$
[u]_{\mathcal{N}_{\infty}^{\beta, q}\left(\mathbb{R}^{n}\right)}:=\sup _{|h|>0}\left\|\frac{\delta_{h} u}{|h|^{\beta}}\right\|_{L^{q}\left(\mathbb{R}^{n}\right)}, \quad 0<\beta \leq 1
$$

and

$$
[u]_{\mathcal{B}_{\infty}^{\beta, q}\left(\mathbb{R}^{n}\right)}:=\sup _{|h|>0}\left\|\frac{\delta_{h}^{2} u}{|h|^{\beta}}\right\|_{L^{q}\left(\mathbb{R}^{n}\right)}, \quad 0<\beta<2 .
$$

This enables us to define the two Besov-type spaces

$$
\mathcal{N}_{\infty}^{\beta, q}\left(\mathbb{R}^{n}\right):=\left\{u \in L^{q}\left(\mathbb{R}^{n}\right) \mid[u]_{\mathcal{N}_{\infty}^{\beta, q}\left(\mathbb{R}^{n}\right)}<\infty\right\}, \quad 0<\beta \leq 1
$$

and

$$
\mathcal{B}_{\infty}^{\beta, q}\left(\mathbb{R}^{n}\right):=\left\{u \in L^{q}\left(\mathbb{R}^{n}\right) \mid[u]_{\mathcal{B}_{\infty}^{\beta, q}\left(\mathbb{R}^{n}\right)}<\infty\right\}, \quad 0<\beta<2 .
$$

The following embedding result can be found in [4, Lemma 2.3].

Lemma 2.4 Let $\beta \in(0,1)$ and $q \in[1, \infty)$. Then we have the continuous embedding

$$
\mathcal{B}_{\infty}^{\beta, q}\left(\mathbb{R}^{n}\right) \hookrightarrow \mathcal{N}_{\infty}^{\beta, q}\left(\mathbb{R}^{n}\right) .
$$

More precisely, for every $u \in \mathcal{B}_{\infty}^{\beta, q}\left(\mathbb{R}^{n}\right)$ we have

$$
[u]_{\mathcal{N}_{\infty}^{\beta, q}\left(\mathbb{R}^{n}\right)} \leq \frac{C}{1-\beta}[u]_{\mathcal{B}_{\infty}^{\beta, q}\left(\mathbb{R}^{n}\right)},
$$

where $C=C(n, q)>0$.

We also need the following embedding result, cf. [2, Theorem 2.8]. 
Lemma 2.5 Let $q \in[1, \infty)$ and $\beta \in(0,1)$ such that $\beta q>n$. If $u \in \mathcal{N}_{\infty}^{\beta, q}\left(\mathbb{R}^{n}\right)$, then for any $\alpha \in(0, \beta-n / q)$ we have $u \in C_{\text {loc }}^{\alpha}\left(\mathbb{R}^{n}\right)$. More precisely, for every $u \in \mathcal{N}_{\infty}^{\beta, q}\left(\mathbb{R}^{n}\right)$ we have

$$
\sup _{\substack{x, y \in \mathbb{R}^{n} \\ x \neq y}} \frac{|u(x)-u(y)|}{|x-y|^{\alpha}} \leq C\left([u]_{\mathcal{N}_{\infty}^{\beta, q}\left(\mathbb{R}^{n}\right)}\right)^{\frac{\alpha q+n}{\beta q}}\left(\|u\|_{L^{q}\left(\mathbb{R}^{n}\right)}\right)^{1-\frac{\alpha q+n}{\beta q}}
$$

where $C=C(n, q, \alpha, \beta)>0$.

Finally, the following result can be found in [1, Proposition 2.6].

Proposition 2.6 Let $s \in(0,1)$.

- Let $0<r<R$. For any function $\psi \in W_{0}^{s, 2}\left(B_{r}\right)$, we have

$$
\sup _{|h|>0}\left\|\frac{\delta_{h} \psi}{|h|^{s}}\right\|_{L^{2}\left(\mathbb{R}^{n}\right)}^{2} \leq C\left(\frac{R}{r}\right)^{n}\left(\frac{R}{R-r}\right)^{3}[\psi]_{W^{s, 2}\left(B_{R}\right)}^{2},
$$

where $C=C(n, s)>0$.

- Let $\Omega \subset \mathbb{R}^{n}$ be an open set and $\psi \in W_{\text {loc }}^{s, 2}(\Omega)$. Then for any $R>0$ such that $B_{R} \Subset \Omega$ and any $0<h_{0} \leq \operatorname{dist}\left(B_{R}, \partial \Omega\right) / 2$, we have

$$
\sup _{|h|>0}\left\|\frac{\delta_{h} \psi}{|h|^{s}}\right\|_{L^{2}\left(B_{R}\right)}^{2} \leq C\|\psi\|_{W^{s, 2}\left(B_{R+h_{0}}\right)}^{2},
$$

where $C=C\left(n, s, R, h_{0}\right)>0$.

\subsection{Some elementary inequalities}

The proof of the following elementary inequality can be found in [2, Lemma A.3].

Lemma 2.7 For all $X, Y \in \mathbb{R}$ and any $p \geq 1$, we have

$$
\left.|| X\right|^{p-1} X-|Y|^{p-1} Y\left|\geq \frac{1}{C}\right| X-\left.Y\right|^{p},
$$

where $C=C(p)>0$.

Next, we prove two elementary inequalities which involve the function $J_{p}$ defined in Sect. 2.1 and are based on the monotonicity property (5) of $\Phi$.

Lemma 2.8 Let $q \geq 1$ and $a, b, c, d \in \mathbb{R}^{n}$. If $\Phi: \mathbb{R} \rightarrow \mathbb{R}$ satisfies (5), then we have

$$
\begin{aligned}
& (\Phi(a-c)-\Phi(b-d))\left(J_{q+1}(a-b)-J_{q+1}(c-d)\right) \\
& \quad \geq \frac{1}{2} \lambda^{-1}|(a-b)-(c-d)|^{2}\left(|a-b|^{q-1}+|c-d|^{q-1}\right) .
\end{aligned}
$$

Proof If $a-c=b-d$, then also $a-b=c-d$, so that in this case both sides of the inequality vanish. Next, we consider the case when $a-c \neq b-d$. In view of the monotonicity assumption (5) imposed on $\Phi$, we have

$$
(\Phi(a-c)-\Phi(b-d))((a-b)-(c-d)) \geq \lambda^{-1}((a-b)-(c-d))^{2} .
$$

Moreover, by [20, page 71], for all $x, y \in \mathbb{R}$ we have

$$
\left(J_{q+1}(y)-J_{q+1}(x)\right)(y-x)=\frac{1}{2}\left(|y|^{q-1}+|x|^{q-1}\right)(y-x)^{2}+\frac{|y|^{q-1}-|x|^{q-1}}{2}\left(y^{2}-x^{2}\right) .
$$


Since the last term on the right-hand side is non-negative, by choosing $y=a-b$ and $x=c-d$ we obtain

$$
\begin{aligned}
& \left(J_{q+1}(a-b)-J_{q+1}(c-d)\right)((a-b)-(c-d)) \\
& \quad \geq \frac{1}{2}\left(|a-b|^{q-1}+|c-d|^{q-1}\right)((a-b)-(c-d))^{2} .
\end{aligned}
$$

Multiplying the inequality (11) with the one in the previous display leads to

$$
\begin{aligned}
& (\Phi(a-c)-\Phi(b-d))\left(J_{q+1}(a-b)-J_{q+1}(c-d)\right)((a-b)-(c-d))^{2} \\
& \geq \frac{1}{2} \lambda^{-1}\left(|a-b|^{q-1}+|c-d|^{q-1}\right)((a-b)-(c-d))^{4},
\end{aligned}
$$

so that the claim follows by simplifying the factor $((a-b)-(c-d))^{2}$ from both sides.

Lemma 2.9 Let $q \geq 1$ and $a, b, c, d \in \mathbb{R}^{n}$. If $\Phi: \mathbb{R} \rightarrow \mathbb{R}$ satisfies (5), then we have

$$
\begin{gathered}
(\Phi(a-c)-\Phi(b-d))\left(J_{q+1}(a-b)-J_{q+1}(c-d)\right) \\
\geq \frac{1}{C}|| a-\left.b\right|^{\frac{q-1}{2}}(a-b)-\left.|c-d|^{\frac{q-1}{2}}(c-d)\right|^{2},
\end{gathered}
$$

where $C=C(\lambda, q)>0$.

Proof If $a-c=b-d$, then both sides of the inequality vanish. Next, let us consider the case when $a-c \neq b-d$. In view of (5), we have

$$
\begin{aligned}
& (\Phi(a-c)-\Phi(b-d))\left(J_{q+1}(a-b)-J_{q+1}(c-d)\right) \\
& =(\Phi(a-c)-\Phi(b-d))((a-c)-(b-d)) \\
& \quad \times\left(J_{q+1}(a-b)-J_{q+1}(c-d)\right)((a-b)-(c-d)) \\
& \quad \times((a-c)-(b-d))^{-2} \\
& \geq \lambda^{-1}\left(J_{q+1}(a-b)-J_{q+1}(c-d)\right)((a-b)-(c-d)) .
\end{aligned}
$$

The right-hand side of the above estimate can be further estimated by applying [2, Lemma A.1] with $p=q+1$ and $q=2$, which yields

$$
\begin{aligned}
& \left(J_{q+1}(a-b)-J_{q+1}(c-d)\right)((a-b)-(c-d)) \\
& \quad \geq q\left(\frac{2}{q+1}\right)^{2}|| a-\left.b\right|^{\frac{q-1}{2}}(a-b)-\left.|c-d|^{\frac{q-1}{2}}(c-d)\right|^{2} .
\end{aligned}
$$

The claim now follows by combining the last two displays.

\subsection{Some preliminary estimates}

The following Caccioppoli-type inequality can be proved in essentially the same way as the one in [18, Theorem 3.1].

Theorem 2.10 Let $0<r<R, x_{0} \in \mathbb{R}^{n}, \lambda \geq 1$ and $f \in L^{\frac{2 n}{n+2 s}}\left(B_{R}\left(x_{0}\right)\right)$. Moreover, assume that $A \in \mathcal{L}_{0}(\lambda)$ and that the Borel function $\Phi: \mathbb{R} \rightarrow \mathbb{R}$ satisfies

$$
|\Phi(t)| \leq \lambda t, \quad \Phi(t) t \geq \lambda^{-1} t^{2} \quad \forall t \in \mathbb{R} .
$$


Then for any local weak solution $u \in W^{s, 2}\left(B_{R}\left(x_{0}\right)\right) \cap L_{2 s}^{1}\left(\mathbb{R}^{n}\right)$ of $L_{A}^{\Phi} u=f$ in $B_{R}\left(x_{0}\right)$, we have

$$
\begin{aligned}
& \int_{B_{r}\left(x_{0}\right)} \int_{B_{r}\left(x_{0}\right)} \frac{|u(x)-u(y)|^{2}}{|x-y|^{n+2 s}} d y d x \\
& \leq C\left(R^{-2 s} \int_{B_{R}\left(x_{0}\right)} u(x)^{2} d x+\int_{\mathbb{R}^{n} \backslash B_{R}\left(x_{0}\right)} \frac{|u(y)|}{\left|x_{0}-y\right|^{n+2 s}} d y \int_{B_{R}\left(x_{0}\right)}|u(x)| d x\right. \\
& \left.\quad+\left(\int_{B_{R}\left(x_{0}\right)}|f(x)|^{\frac{2 n}{n+2 s}} d x\right)^{\frac{n+2 s}{n}}\right)
\end{aligned}
$$

where $C=C(n, s, \lambda, r, R)>0$.

We remark that the assumptions in (12) are clearly implied by the assumptions $\Phi(0)=0$, (4) and (5) which are used in our main results.

The following result on local boundedness is essentially given by [3, Theorem 3.8], where the below result is stated under the stronger assumption that $u \in W_{0}^{s, 2}\left(B_{R}\left(x_{0}\right)\right)$ and in setting of the fractional $p$-Laplacian, which applied to our setting means that strictly speaking it only contains the case when $\Phi(t)=t$ and $A(x, y) \equiv 1$. Nevertheless, an inspection of the proof shows that it remains valid for local weak solutions, see also [2, Theorem 3.2]. Moreover, the case of a general $\Phi$ and a general $A$ can easily be treated by noting that the Caccioppoli-type inequality from [3, Proposition 3.5] remains valid for such a general $\Phi$ and a general $A$ by simply applying the bounds imposed on $\Phi$ and $A$ whenever appropriate in a similar fashion as in [18, Theorem 3.1]. Therefore, we have the following result.

Theorem 2.11 Let $R>0, x_{0} \in \mathbb{R}^{n}, \lambda \geq 1, \sigma \in(0,1)$ and $f \in L^{q}\left(B_{R}\left(x_{0}\right)\right)$ for some $q>\frac{n}{2 s}$. Moreover, consider a kernel coefficient $A \in \mathcal{L}_{0}(\lambda)$ and assume that the Borel function $\Phi: \mathbb{R} \rightarrow \mathbb{R}$ satisfies (12). Then for any local weak solution $u \in W^{s, 2}\left(B_{R}\left(x_{0}\right)\right) \cap L_{2 s}^{1}\left(\mathbb{R}^{n}\right)$ of the equation

$$
L_{A}^{\Phi} u=f \text { in } B_{R}\left(x_{0}\right)
$$

we have the estimate

$$
\begin{aligned}
\sup _{x \in \bar{B}_{\sigma R}\left(x_{0}\right)}|u(x)| \leq & C\left(\left(f_{B_{R}\left(x_{0}\right)} u(x)^{2} d x\right)^{\frac{1}{2}}+R^{2 s} \int_{\mathbb{R}^{n} \backslash B_{\sigma R}\left(x_{0}\right)} \frac{|u(y)|}{\left|x_{0}-y\right|^{n+2 s}} d y\right. \\
& \left.+R^{2 s-\frac{n}{q}}\|f\|_{L^{q}\left(B_{R}\left(x_{0}\right)\right)}\right),
\end{aligned}
$$

where $C=C(n, s, \lambda, q, \sigma)>0$.

In the case when $f=0$ and $\Phi(t)=t$, the following result concerning basic Hölder regularity follows from [8, Theorem 1.2]. The case of a general $\Phi$ can again be treated by replacing the Caccioppoli inequality given by [8, Theorem 1.4] with the one from [18, Theorem 3.1]. The result with a general right-hand side can then be proved in essentially the same way as in [2, section 3.2].

Theorem 2.12 Under the same assumptions and notation as in Theorem 2.11, there exists some $\beta=\beta(n, s, \lambda, q, \sigma) \in(0,1)$ such that $u \in C^{\beta}\left(\bar{B}_{\sigma R}\left(x_{0}\right)\right)$. Moreover, we have the estimate 


$$
\begin{aligned}
{[u]_{C^{\beta}\left(B_{\sigma R}\left(x_{0}\right)\right)} \leq } & C\left(\left(f_{B_{R}\left(x_{0}\right)} u(x)^{2} d x\right)^{\frac{1}{2}}+R^{2 s} \int_{\mathbb{R}^{n} \backslash B_{\sigma R}\left(x_{0}\right)} \frac{|u(y)|}{\left|x_{0}-y\right|^{n+2 s}} d y\right. \\
& \left.+R^{2 s-\frac{n}{q}}\|f\|_{L^{q}\left(B_{R}\left(x_{0}\right)\right)}\right)
\end{aligned}
$$

where $C=C(n, s, \lambda, q, \sigma, \beta)>0$.

\section{Higher Hölder regularity for homogeneous equations with locally translation invariant kernel}

\subsection{Incremental higher integrability and differentiability}

The key ingredient to proving the desired higher Hölder regularity for homogeneous equations with locally translation invariant kernel is provided by the following incremental higher integrability and differentiability result on the scale of Besov-type spaces. In the case of the fractional $p$-Laplacian for $p \geq 2$, the below result was proved in [2, Proposition 5.1]. Besides the fact that we treat equations with arbitrary locally translation invariant kernels, it is also interesting that in our setting of equations with linear growth, we are able to directly prove both higher integrability and differentiability, while for possibly degenerate equations as in [2] it is necessary to first obtain a pure higher integrability result (cf. [2, Proposition 4.1]), which is then used in order to also obtain higher differentiability. We remark that this additional higher differentiability does not seem to have a counterpart in the context of local equations and is one of the main reasons why in our nonlocal setting we are able to exceed $C^{s}$ regularity.

Moreover, note that although at this point we work with solutions that are bounded, this assumption will later be removed by using Theorem 2.11 .

Proposition 3.1 Let $u \in W^{s, 2}\left(B_{1}\right) \cap L_{2 s}^{1}\left(\mathbb{R}^{n}\right) \cap L^{\infty}\left(B_{1}\right)$ be a local weak solution of

$$
L_{A}^{\Phi} u=0 \text { in } B_{1},
$$

where $A \in \mathcal{L}_{1}\left(\lambda, B_{1}\right)$ and $\Phi$ satisfies (4) and (5). Suppose that

$$
\|u\|_{L^{\infty}\left(B_{1}\right)} \leq 1, \quad \int_{\mathbb{R}^{n} \backslash B_{1}} \frac{|u(y)|}{|y|^{n+2 s}} d y \leq 1,
$$

and that for some $q \geq 2, \vartheta \in \mathbb{R}$ such that $0<(1+\vartheta q) / q<1$ and some $0<h_{0}<1$, we have

$$
\sup _{0<|h|<h_{0}}\left\|\frac{\delta_{h}^{2} u}{|h|^{\frac{1+\vartheta q}{q}}}\right\|_{L^{q}\left(B_{1}\right)}^{q}<+\infty .
$$

Then for any radius $4 h_{0}<R \leq 1-2 h_{0}$, we have

$$
\sup _{0<|h|<h_{0}}\left\|\frac{\delta_{h}^{2} u}{|h|^{\frac{1+2 s+\vartheta q}{q+1}}}\right\|_{L^{q+1}\left(B_{R-4 h_{0}}\right)}^{q+1} \leq C\left(\sup _{0<|h|<h_{0}}\left\|\frac{\delta_{h}^{2} u}{|h|^{\frac{1+\vartheta q}{q}}}\right\|_{L^{q}\left(B_{R+4 h_{0}}\right)}^{q}+1\right) .
$$

where $C=C\left(n, s, q, \lambda, h_{0}\right)>0$. 
Proof Step 1: Discrete differentiation of the equation. Set $r:=R-4 h_{0}>0$ and fix some $h \in \mathbb{R}^{n}$ such that $0<|h|<h_{0}$. Let $\eta \in C_{0}^{\infty}\left(B_{R}\right)$ be a non-negative Lipschitz cutoff function satisfying

$$
\eta \equiv 1 \text { in } B_{r}, \quad \eta \equiv 0 \text { in } \mathbb{R}^{n} \backslash B_{(R+r) / 2}, \quad|\nabla \eta| \leq \frac{C_{1}}{R-r}=\frac{C_{1}}{4 h_{0}} .
$$

Let us show that the function

$$
\varphi=J_{q+1}\left(\frac{\delta_{h} u}{|h|^{\vartheta}}\right) \eta^{2}=\left|\frac{\delta_{h} u}{|h|^{\vartheta}}\right|^{q-1} \frac{\delta_{h} u}{|h|^{\vartheta}} \eta^{2}
$$

belongs to $W^{s, 2}\left(B_{R}\right)$. Since $\|u\|_{L^{\infty}\left(B_{1}\right)} \leq 1$ implies $\|u\|_{L^{\infty}\left(B_{R}\right)} \leq 1$ and also $\left\|u_{h}\right\|_{L^{\infty}\left(B_{R}\right)} \leq$ 1, we have $\|\varphi\|_{L^{\infty}\left(B_{R}\right)} \leq \frac{2^{q}}{|h|^{\vartheta}}$ and therefore $\varphi \in L^{\infty}\left(B_{R}\right) \subset L^{2}\left(B_{R}\right)$. Moreover, note that the function $t \mapsto J_{q+1}(t)$ is Lipschitz continuous on the domain $t \in[-2,2]$ with Lipschitz constant $q 2^{q-1}$. Therefore, since we have $\left\|\delta_{h} u\right\|_{L^{\infty}\left(B_{R}\right)} \leq 2$, we obtain

$$
\begin{aligned}
\int_{B_{R}} \int_{B_{R}} \frac{\left|J_{q+1}\left(\delta_{h} u(x)\right)-J_{q+1}\left(\delta_{h} u(y)\right)\right|^{2}}{|x-y|^{n+2 s}} d y d x & \leq C_{2} \int_{B_{R}} \int_{B_{R}} \frac{\left|\delta_{h} u(x)-\delta_{h} u(y)\right|^{2}}{|x-y|^{n+2 s}} d y d x \\
& \leq 2 C_{2}\left(\left[u_{h}\right]_{W^{s, 2}\left(B_{R}\right)}^{2}+[u]_{W^{s, 2}\left(B_{R}\right)}^{2}\right)<\infty,
\end{aligned}
$$

where $C_{2}=C_{2}(q)>0$, so that $J_{q+1}\left(\delta_{h} u\right) \in W^{s, 2}\left(B_{R}\right)$. Thus, since the product of a function belonging to $W^{s, 2}\left(B_{R}\right)$ and a Lipschitz function also belongs to $W^{s, 2}\left(B_{R}\right)$ (cf. [9, Lemma 5.3]), $\varphi=J_{q+1}\left(\delta_{h} u\right) \frac{\eta^{2}}{|h|^{\vartheta q}}$ also belongs to $W^{s, 2}\left(B_{R}\right)$.

Next, consider the function $\varphi_{-h}(x):=\varphi(x-h)$. Since both $\varphi$ and $\varphi_{-h}$ belong to $W^{s, 2}\left(B_{R-h_{0}}\right)$ and are compactly supported in $B_{R-h_{0}}$, in view of [2, Lemma 2.11] in particular both $\varphi$ and $\varphi_{-h}$ belong to $W_{c}^{s, 2}\left(B_{1}\right)$, so that both $\varphi$ and $\varphi_{-h}$ are admissible test functions in (13). Therefore, using $\varphi_{-h}$ as a test function in (13) along with a change of variables yields

$$
\begin{aligned}
0 & =\int_{\mathbb{R}^{n}} \int_{\mathbb{R}^{n}} \frac{A(x, y)}{|x-y|^{n+2 s}} \Phi(u(x)-u(y))\left(\varphi_{-h}(x)-\varphi_{-h}(y)\right) d y d x \\
& =\int_{\mathbb{R}^{n}} \int_{\mathbb{R}^{n}} \frac{A_{h}(x, y)}{|x-y|^{n+2 s}} \Phi\left(u_{h}(x)-u_{h}(y)\right)(\varphi(x)-\varphi(y)) d y d x,
\end{aligned}
$$

where we have set $A_{h}(x, y):=A(x+h, y+h)$. Moreover, testing (13) with $\varphi$ yields

$$
\int_{\mathbb{R}^{n}} \int_{\mathbb{R}^{n}} \frac{A(x, y)}{|x-y|^{n+2 s}} \Phi(u(x)-u(y))(\varphi(x)-\varphi(y)) d y d x=0 .
$$

By subtracting (16) from (15) and dividing by $0<|h|<h_{0}$, we obtain

$$
\int_{\mathbb{R}^{n}} \int_{\mathbb{R}^{n}} \frac{A_{h}(x, y) \Phi\left(u_{h}(x)-u_{h}(y)\right)-A(x, y) \Phi(u(x)-u(y))}{|h||x-y|^{n+2 s}}(\varphi(x)-\varphi(y)) d y d x=0 .
$$

Next, splitting the above integral and taking into account the choice of $\varphi$, we arrive at

$$
I_{1}+I_{2}+I_{3}=0,
$$

where

$$
\begin{aligned}
I_{1}:= & \int_{B_{R}} \int_{B_{R}} \frac{A_{h}(x, y) \Phi\left(u_{h}(x)-u_{h}(y)\right)-A(x, y) \Phi(u(x)-u(y))}{|h|^{1+\vartheta q}|x-y|^{n+2 s}} \\
& \times\left(J_{q+1}\left(u_{h}(x)-u(x)\right) \eta(x)^{2}-J_{q+1}\left(u_{h}(y)-u(y)\right) \eta(y)^{2}\right) d y d x,
\end{aligned}
$$




$$
\begin{aligned}
I_{2}:= & \int_{B_{\frac{R+r}{2}}} \int_{\mathbb{R}^{n} \backslash B_{R}} \frac{A_{h}(x, y) \Phi\left(u_{h}(x)-u_{h}(y)\right)-A(x, y) \Phi(u(x)-u(y))}{|h|^{1+\vartheta q}|x-y|^{n+2 s}} \\
& \times J_{q+1}\left(u_{h}(x)-u(x)\right) \eta(x)^{2} d y d x, \\
I_{3}:= & -\int_{\mathbb{R}^{n} \backslash B_{R}} \int_{B_{\frac{R+r}{2}}} \frac{A_{h}(x, y) \Phi\left(u_{h}(x)-u_{h}(y)\right)-A(x, y) \Phi(u(x)-u(y))}{|h|^{1+\vartheta q|x-y|^{n+2 s}}} \\
& \times J_{q+1}\left(u_{h}(y)-u(y)\right) \eta(y)^{2} d y d x,
\end{aligned}
$$

where we used that $\eta$ vanishes identically outside of $B_{(R+r) / 2}$.

Step 2: Preliminary estimation of the local term $I_{1}$. Since $A \in \mathcal{L}_{1}\left(\lambda, B_{1}\right)$, we have $A(x, y)=a(x-y)$ for all $x, y \in B_{1}$ and some measurable function $a: \mathbb{R}^{n} \rightarrow \mathbb{R}$. Since for $x, y \in B_{R}$ we have $x+h, y+h \in B_{1}$, it follows that for all $x, y \in B_{R}$ we have

$$
\left.A_{h}(x, y)=A(x+h, y+h)=a((x+h)-(y+h))\right)=a(x-y)=A(x, y) .
$$

Therefore, we can rewrite $I_{1}$ as follows

$$
\begin{aligned}
I_{1}= & \int_{B_{R}} \int_{B_{R}} \frac{A(x, y)\left(\Phi\left(u_{h}(x)-u_{h}(y)\right)-\Phi(u(x)-u(y))\right)}{|h|^{1+\vartheta q}|x-y|^{n+2 s}} \\
& \times\left(J_{q+1}\left(u_{h}(x)-u(x)\right) \eta(x)^{2}-J_{q+1}\left(u_{h}(y)-u(y)\right) \eta(y)^{2}\right) d y d x .
\end{aligned}
$$

Let us now concentrate on estimating $I_{1}$. First of all, we observe that

$$
\begin{aligned}
& J_{q+1}\left(u_{h}(x)-u(x)\right) \eta(x)^{2}-J_{q+1}\left(u_{h}(y)-u(y)\right) \eta(y)^{2} \\
& =\frac{\left(J_{q+1}\left(u_{h}(x)-u(x)\right)-J_{q+1}\left(u_{h}(y)-u(y)\right)\right)}{2}\left(\eta(x)^{2}+\eta(y)^{2}\right) \\
& \quad+\frac{\left(J_{q+1}\left(u_{h}(x)-u(x)\right)+J_{q+1}\left(u_{h}(y)-u(y)\right)\right)}{2}\left(\eta(x)^{2}-\eta(y)^{2}\right) .
\end{aligned}
$$

Therefore, we obtain

$$
\begin{aligned}
& \left(\Phi\left(u_{h}(x)-u_{h}(y)\right)-\Phi(u(x)-u(y))\right) \\
& \quad\left(J_{q+1}\left(u_{h}(x)-u(x)\right) \eta(x)^{2}-J_{q+1}\left(u_{h}(y)-u(y)\right) \eta(y)^{2}\right) \\
& \geq\left(\Phi\left(u_{h}(x)-u_{h}(y)\right)-\Phi(u(x)-u(y))\right) \\
& \quad \times\left(J_{q+1}\left(u_{h}(x)-u(x)\right)-J_{q+1}\left(u_{h}(y)-u(y)\right)\right) \frac{\left(\eta(x)^{2}+\eta(y)^{2}\right)}{2} \\
& \quad-\left|\Phi\left(u_{h}(x)-u_{h}(y)\right)-\Phi(u(x)-u(y))\right|\left(\left|u_{h}(x)-u(x)\right|^{q}\right. \\
& \left.\quad+\left|u_{h}(y)-u(y)\right|^{q}\right)\left|\frac{\eta(x)^{2}-\eta(y)^{2}}{2}\right| .
\end{aligned}
$$

Next, using the Lipschitz bound (4), Young's inequality and then Lemma 2.8, for the negative term in the last display we deduce

$$
\begin{aligned}
\mid \Phi & \left(u_{h}(x)-u_{h}(y)\right)-\Phi(u(x)-u(y)) \mid\left(\left|u_{h}(x)-u(x)\right|^{q}\right. \\
+ & \left.\left|u_{h}(y)-u(y)\right|^{q}\right)\left|\frac{\eta(x)^{2}-\eta(y)^{2}}{2}\right| \\
\leq & \frac{\lambda}{2}\left|\left(u_{h}(x)-u_{h}(y)\right)-(u(x)-u(y))\right| \\
& \times\left(\left|u_{h}(x)-u(x)\right|^{\frac{q-1}{2}}\left|u_{h}(x)-u(x)\right|^{\frac{q+1}{2}}+\left|u_{h}(y)-u(y)\right|^{\frac{q-1}{2}}\left|u_{h}(y)-u(y)\right|^{\frac{q+1}{2}}\right)
\end{aligned}
$$




$$
\begin{aligned}
& \times(\eta(x)+\eta(y))|\eta(x)-\eta(y)| \\
\leq & \frac{\lambda}{4 \varepsilon}\left(\left|u_{h}(x)-u(x)\right|^{q+1}+\left|u_{h}(y)-u(y)\right|^{q+1}\right)|\eta(x)-\eta(y)|^{2} \\
& +\frac{\lambda}{2} \varepsilon\left|\left(u_{h}(x)-u_{h}(y)\right)-(u(x)-u(y))\right|^{2}\left(\left|u_{h}(x)-u(x)\right|^{q-1}+\left|u_{h}(y)-u(y)\right|^{q-1}\right) \\
& \times\left(\eta(x)^{2}+\eta(y)^{2}\right) \\
\leq & \frac{\lambda}{4 \varepsilon}\left(\left|u_{h}(x)-u(x)\right|^{q+1}+\left|u_{h}(y)-u(y)\right|^{q+1}\right)|\eta(x)-\eta(y)|^{2} \\
& +\lambda^{2} \varepsilon\left(\Phi\left(u_{h}(x)-u_{h}(y)\right)-\Phi(u(x)-u(y))\right) \\
& \times\left(J_{q+1}\left(u_{h}(x)-u(x)\right)-J_{q+1}\left(u_{h}(y)-u(y)\right)\right)\left(\eta(x)^{2}+\eta(y)^{2}\right),
\end{aligned}
$$

where $\varepsilon>0$ is arbitrary. By choosing $\varepsilon:=\frac{1}{2 \lambda^{2}}$, combining the last two displays yields

$$
\begin{aligned}
I_{1} \geq & \frac{1}{4} \int_{B_{R}} \int_{B_{R}} \frac{A(x, y)\left(\Phi\left(u_{h}(x)-u_{h}(y)\right)-\Phi(u(x)-u(y))\right)}{|h|^{1+\vartheta q}|x-y|^{n+2 s}} \\
& \times\left(J_{q+1}\left(u_{h}(x)-u(x)\right)-J_{q+1}\left(u_{h}(y)-u(y)\right)\right)\left(\eta(x)^{2}+\eta(y)^{2}\right) d y d x \\
& -C_{3} \int_{B_{R}} \int_{B_{R}} \frac{A(x, y)\left(\left|u_{h}(x)-u(x)\right|^{q+1}+\left|u_{h}(y)-u(y)\right|^{q+1}\right)|\eta(x)-\eta(y)|^{2}}{|h|^{1+\vartheta q}|x-y|^{n+2 s}} d y d x,
\end{aligned}
$$

where $C_{3}=C_{3}(\lambda)>0$. By using Lemma 2.9, we can further estimate the first term of the previous display, which along with the bounds (2) of $A$ leads to

$$
\begin{aligned}
I_{1} \geq & c \int_{B_{R}} \int_{B_{R}}\left|\frac{\left|\delta_{h} u(x)\right|^{\frac{q-1}{2}} \delta_{h} u(x)}{|h|^{\frac{1+\vartheta q}{2}}}-\frac{\left|\delta_{h} u(y)\right|^{\frac{q-1}{2}} \delta_{h} u(y)}{|h|^{\frac{1+\vartheta q}{2}}}\right|^{2} \frac{\eta(x)^{2}+\eta(y)^{2}}{|x-y|^{n+2 s}} d y d x \\
& -C_{4} \int_{B_{R}} \int_{B_{R}} \frac{\left(\left|\delta_{h} u(x)\right|^{q+1}+\left|\delta_{h} u(y)\right|^{q+1}\right)|\eta(x)-\eta(y)|^{2}}{|h|^{1+\vartheta q}|x-y|^{n+2 s}} d y d x,
\end{aligned}
$$

where $c=c(\lambda, q)>0$ and $C_{4}=C_{4}(\lambda)>0$. Next, for simplicity we write

$$
X:=\frac{\left|\delta_{h} u(x)\right|^{\frac{q-1}{2}} \delta_{h} u(x)}{|h|^{\frac{1+\vartheta q}{2}}} \text { and } Y:=\frac{\left|\delta_{h} u(y)\right|^{\frac{q-1}{2}} \delta_{h} u(y)}{|h|^{\frac{1+\vartheta q}{2}}}
$$

and observe that by using the convexity of the function $t \mapsto t^{2}$, we obtain

$$
\begin{aligned}
|X \eta(x)-Y \eta(y)|^{2} & =\left|(X-Y) \frac{\eta(x)+\eta(y)}{2}+(X+Y) \frac{\eta(x)-\eta(y)}{2}\right|^{2} \\
& \leq \frac{1}{2}|X-Y|^{2}|\eta(x)+\eta(y)|^{2}+\frac{1}{2}|X+Y|^{2}|\eta(x)-\eta(y)|^{2} \\
& \leq|X-Y|^{2}\left(\eta(x)^{2}+\eta(y)^{2}\right)+\left(X^{2}+Y^{2}\right)|\eta(x)-\eta(y)|^{2} .
\end{aligned}
$$

Combining (18) with the last display yields

$$
\begin{aligned}
I_{1} \geq & c \int_{B_{R}} \int_{B_{R}}\left|\frac{\left|\delta_{h} u(x)\right|^{\frac{q-1}{2}} \delta_{h} u(x)}{|h|^{\frac{1+\vartheta q}{2}}} \eta(x)-\frac{\left|\delta_{h} u(y)\right|^{\frac{q-1}{2}} \delta_{h} u(y)}{|h|^{\frac{1+\vartheta q}{2}}} \eta(y)\right|^{2} \frac{1}{|x-y|^{n+2 s}} d y d x \\
& -c \int_{B_{R}} \int_{B_{R}}\left(\frac{\left|\delta_{h} u(x)\right|^{q+1}}{|h|^{1+\vartheta q}}+\frac{\left|\delta_{h} u(y)\right|^{q+1}}{|h|^{1+\vartheta q}}\right) \frac{|\eta(x)-\eta(y)|^{2}}{|x-y|^{n+2 s}} d y d x
\end{aligned}
$$




$$
\begin{aligned}
& -C_{4} \int_{B_{R}} \int_{B_{R}} \frac{\left(\left|\delta_{h} u(x)\right|^{q+1}+\left|\delta_{h} u(y)\right|^{q+1}\right)|\eta(x)-\eta(y)|^{2}}{|h|^{1+\vartheta q}|x-y|^{n+2 s}} d y d x \\
= & c\left[\frac{\left|\delta_{h} u\right|^{\frac{q-1}{2}} \delta_{h} u}{\left.|h|^{\frac{1+\vartheta q}{2}} \eta\right]_{W^{s, 2}\left(B_{R}\right)}^{2}}\right. \\
& -C_{5} \int_{B_{R}} \int_{B_{R}}\left(\frac{\left|\delta_{h} u(x)\right|^{q+1}}{|h|^{1+\vartheta q}}+\frac{\left|\delta_{h} u(y)\right|^{q+1}}{|h|^{1+\vartheta q}}\right) \frac{|\eta(x)-\eta(y)|^{2}}{|x-y|^{n+2 s}} d y d x,
\end{aligned}
$$

where $C_{5}=C_{5}(\lambda, q)>0$. By combining the above estimate for $I_{1}$ with the identity $I_{1}+I_{2}+I_{3}=0$, we arrive at

$$
\left[\frac{\left|\delta_{h} u\right|^{\frac{q-1}{2}} \delta_{h} u}{|h|^{\frac{1+\vartheta q}{2}} \eta}\right]_{W^{s, 2}\left(B_{R}\right)}^{2} \leq C_{6}\left(I_{1,1}+\left|I_{2}\right|+\left|I_{3}\right|\right),
$$

where $C_{6}=C_{6}(\lambda, q)>0$ and

$$
I_{1,1}:=\int_{B_{R}} \int_{B_{R}}\left(\frac{\left|\delta_{h} u(x)\right|^{q+1}}{|h|^{1+\vartheta q}}+\frac{\left|\delta_{h} u(y)\right|^{q+1}}{|h|^{1+\vartheta q}}\right) \frac{|\eta(x)-\eta(y)|^{2}}{|x-y|^{n+2 s}} d y d x .
$$

Our next goal is to estimate the terms $I_{1,1},\left|I_{2}\right|$ and $\left|I_{3}\right|$.

Step 3: Estimating the local term $I_{1,1}$. In order to estimate $I_{1,1}$, observe that for any $x \in B_{R}$ changing variables and integrating in polar coordinates yields

$$
\int_{B_{R}} \frac{d y}{|x-y|^{n+2 s-2}} \leq \int_{B_{2 R}} \frac{d z}{|z|^{n+2 s-2}}=C_{7} R^{2-2 s} \leq C_{7},
$$

where $C_{7}=C_{7}(n, s)>0$. Since by construction $\eta$ is Lipschitz with Lipschitz constant $\frac{C_{1}}{4 h_{0}}$, along with (20) we obtain

$$
\begin{aligned}
& \int_{B_{R}} \int_{B_{R}} \frac{\left|\delta_{h} u(x)\right|^{q+1}}{|h|^{1+\vartheta q}} \frac{|\eta(x)-\eta(y)|^{2}}{|x-y|^{n+2 s}} d y d x \\
& \leq\left(\frac{C_{1}}{4 h_{0}}\right)^{2} \int_{B_{R}}\left(\int_{B_{R}} \frac{d y}{|x-y|^{n+2 s-2}}\right) \frac{\left|\delta_{h} u(x)\right|^{q+1}}{|h|^{1+\vartheta q}} d x \\
& \leq C_{7}\left(\frac{C_{1}}{4 h_{0}}\right)^{2} \int_{B_{R}} \frac{\left|\delta_{h} u(x)\right|^{q+1}}{|h|^{1+\vartheta q}} d x \\
& \leq C_{8}|| u||_{L^{\infty}\left(B_{R+h_{0}}\right)} \int_{B_{R}} \frac{\left|\delta_{h} u(x)\right|^{q}}{|h|^{1+\vartheta q}} d x \\
& \quad \leq C_{8} \int_{B_{R}} \frac{\left|\delta_{h} u(x)\right|^{q}}{|h|^{1+\vartheta q}} d x,
\end{aligned}
$$

where we used that $R+h_{0} \leq 1$ and $\|u\|_{L^{\infty}\left(B_{1}\right)} \leq 1$ in order to obtain the last inequality and $C_{8}=C_{8}\left(n, s, q, \lambda, h_{0}\right)>0$. In the same way we have

$$
\int_{B_{R}} \int_{B_{R}} \frac{\left|\delta_{h} u(y)\right|^{q+1}}{|h|^{1+\vartheta q}} \frac{|\eta(x)-\eta(y)|^{2}}{|x-y|^{n+2 s}} d y d x \leq C_{8} \int_{B_{R}} \frac{\left|\delta_{h} u(y)\right|^{q}}{|h|^{1+\vartheta q}} d y,
$$

so that we obtain

$$
I_{1,1} \leq 2 C_{8} \int_{B_{R}} \frac{\left|\delta_{h} u(x)\right|^{q}}{|h|^{1+\vartheta q}} d x
$$


Step 4: Estimating the nonlocal terms $I_{2}$ and $I_{3}$. Next, let us estimate the nonlocal terms $I_{2}$ and $I_{3}$, which can be treated in the same way. Since $\|u\|_{L^{\infty}\left(B_{1}\right)} \leq 1$ and $(R+r) / 2+h_{0} \leq 1$, by additionally using the bound (4) of $\Phi$ with $t=u_{h}(x)-u_{h}(y)$ and $t^{\prime}=0$, for almost every $x \in B_{(R-r) / 2}$ and any $y \in \mathbb{R}^{n} \backslash B_{R}$ we have

$$
\begin{aligned}
\left|\Phi\left(u_{h}(x)-u_{h}(y)\right) J_{q+1}\left(\delta_{h} u(x)\right)\right| & \leq \lambda\left(\left.|| u\right|_{L^{\infty}\left(B_{(R+r) / 2+h_{0}}\right)}+\left|u_{h}(y)\right|\right)\left|\delta_{h} u(x)\right|^{q} \\
& \leq \lambda\left(1+\left|u_{h}(y)\right|\right)\left|\delta_{h} u(x)\right|^{q}
\end{aligned}
$$

and similarly

$$
\left|\Phi(u(x)-u(y)) J_{q+1}\left(\delta_{h} u(x)\right)\right| \leq \lambda(1+|u(y)|)\left|\delta_{h} u(x)\right|^{q} .
$$

By using the upper bound in (2) of $A$ (which trivially also holds for $A_{h}$ ) and the fact that $0 \leq \eta \leq 1$ and then the last two displays, we deduce

$$
\begin{aligned}
\left|I_{2}\right| & \leq \lambda \int_{B_{\frac{R+r}{2}}} \int_{\mathbb{R}^{n} \backslash B_{R}} \frac{\left(\left|\Phi\left(u_{h}(x)-u_{h}(y)\right)\right|+|\Phi(u(x)-u(y))|\right)\left|J_{q+1}\left(\delta_{h} u(x)\right)\right|}{|h|^{1+\vartheta q}|x-y|^{n+2 s}} d y d x \\
& \leq 2 \lambda^{2} \int_{B_{\frac{R+r}{2}}} \int_{\mathbb{R}^{n} \backslash B_{R}} \frac{\left(1+\left|u_{h}(y)\right|+|u(y)|\right)\left|\delta_{h} u(x)\right|^{q}}{|h|^{1+\vartheta q}|x-y|^{n+2 s}} d y d x .
\end{aligned}
$$

For any $x \in B_{(R+r) / 2)}$, we have $B_{(R-r) / 2}(x) \subset B_{R}$, which in view of integration in polar coordinates along with the fact that $R-r=4 h_{0}$ leads to

$$
\begin{aligned}
& \int_{\mathbb{R}^{n} \backslash B_{R}} \frac{d y}{|x-y|^{n+2 s}} \leq \int_{\mathbb{R}^{n} \backslash B_{\frac{R-r}{2}}(x)} \frac{d y}{|x-y|^{n+2 s}}=\int_{\mathbb{R}^{n} \backslash B_{\frac{R-r}{2}}} \frac{d z}{|z|^{n+2 s}}=C_{9}\left(\frac{R-r}{2}\right)^{-2 s} \\
& \quad=C_{10},
\end{aligned}
$$

where $C_{9}=C_{9}(n, s)>0$ and $C_{10}=C_{9}\left(2 h_{0}\right)^{-2 s}$. Using Lemma 2.1, the change of variables $z=y+h$ and then Lemma 2.2, for any $x \in B_{(R+r) / 2}$ we obtain

$$
\begin{aligned}
\int_{\mathbb{R}^{n} \backslash B_{R}} \frac{\left|u_{h}(y)\right|}{|x-y|^{n+2 s}} d y & \leq\left(\frac{2 R}{R-r}\right)^{n+2 s} \int_{\mathbb{R}^{n} \backslash B_{R}} \frac{\left|u_{h}(y)\right|}{|y|^{n+2 s}} d y \\
& \leq\left(2 h_{0}\right)^{-(n+2 s)} \int_{\mathbb{R}^{n} \backslash B_{R}(h)} \frac{|u(z)|}{|h-z|^{n+2 s}} d z \\
& \leq\left(2 h_{0} R\right)^{-(n+2 s)}\left(\left.|| u\right|_{L^{1}\left(B_{1}\right)}+\int_{\mathbb{R}^{n} \backslash B_{1}} \frac{|u(z)|}{|z|^{n+2 s}} d z\right) \\
& \leq\left(8 h_{0}^{2}\right)^{-(n+2 s)}\left(\|u\|_{L^{\infty}\left(B_{1}\right)}\left|B_{1}\right|+\int_{\mathbb{R}^{n} \backslash B_{1}} \frac{|u(z)|}{|z|^{n+2 s}} d z\right) \leq C_{11},
\end{aligned}
$$

where $C_{11}=C_{11}\left(n, s, h_{0}\right)>0$. Here we also used the the fact that $R>4 h_{0}$ and the bounds imposed on $u$. The term involving $u$ can be estimated similarly. In fact, by using Lemma 2.1 and Lemma 2.2, for any $x \in B_{(R+r) / 2}$ we obtain

$$
\begin{aligned}
\int_{\mathbb{R}^{n} \backslash B_{R}} \frac{|u(y)|}{|x-y|^{n+2 s}} d y & \leq\left(2 h_{0}\right)^{-(n+2 s)} \int_{\mathbb{R}^{n} \backslash B_{R}} \frac{|u(y)|}{|y|^{n+2 s}} d y \\
& \leq\left(8 h_{0}^{2}\right)^{-(n+2 s)}\left(\|\left. u\right|_{L^{\infty}\left(B_{1}\right)}\left|B_{1}\right|+\int_{\mathbb{R}^{n} \backslash B_{1}} \frac{|u(y)|}{|y|^{n+2 s}} d y\right) \leq C_{12},
\end{aligned}
$$


where $C_{12}=C_{12}\left(n, s, h_{0}\right)>0$. By combining the above estimates with (22) and the observation that $\left|I_{3}\right|$ can be estimated in the same way, we arrive at

$$
\left|I_{2}\right|+\left|I_{3}\right| \leq C_{13} \int_{B_{\frac{R+r}{2}}} \frac{\left|\delta_{h} u(x)\right|^{q}}{|h|^{1+\vartheta q}} d x \leq C_{13} \int_{B_{R}} \frac{\left|\delta_{h} u(x)\right|^{q}}{|h|^{1+\vartheta q}} d x,
$$

where $C_{13}=C_{13}\left(n, s, \lambda, h_{0}\right)>0$. By combining this estimate with (21) and (19), we find the estimate

$$
\left[\frac{\left|\delta_{h} u\right|^{\frac{q-1}{2}} \delta_{h} u}{|h|^{\frac{1+\vartheta q}{2}} \eta}\right]_{W^{s, 2}\left(B_{R}\right)}^{2} \leq C_{14} \int_{B_{R}} \frac{\left|\delta_{h} u(x)\right|^{q}}{|h|^{1+\vartheta q}} d x,
$$

where $C_{14}=C_{14}\left(n, s, q, \lambda, h_{0}\right)>0$.

Step 5: Conclusion. Let $\xi \in \mathbb{R}^{n} \backslash\{0\}$ to be chosen such that $|\xi|<h_{0}$. Applying Lemma 2.7 with

$$
X=u(x+h+\xi)-u(x+\xi), \quad Y=u(x+h)-u(x), \quad p=\frac{q+1}{2}
$$

leads to

$$
\begin{aligned}
\left\|\frac{\delta_{\xi} \delta_{h} u}{|\xi|^{\frac{2 s}{q+1}}|h|^{\frac{1+\vartheta q}{q+1}}}\right\|\left\|_{L^{q+1}\left(B_{r}\right)}^{q+1} \leq C_{15}\right\| \frac{\delta_{\xi}\left(\left|\delta_{h} u\right|^{\frac{q-1}{2}} \delta_{h} u\right)}{|\xi|^{s}|h|^{\frac{1+\vartheta q}{2}}} \|_{L^{2}\left(B_{r}\right)}^{2} \\
\leq C_{15}\left\|\eta \frac{\delta_{\xi}}{|\xi|^{s}}\left(\frac{\left|\delta_{h} u\right|^{\frac{q-1}{2}} \delta_{h} u}{|h|^{\frac{1+\vartheta q}{2}}}\right)\right\|_{L^{2}\left(\mathbb{R}^{n}\right)}^{2},
\end{aligned}
$$

where $C_{15}=C_{15}(q)>0$. Here we also used that $\eta \equiv 1$ in $B_{r}$ in order to obtain the last inequality. Next, we observe that by the discrete Leibniz rule (cf. [2, Formula (2.1)]), we can write

$$
\eta \delta_{\xi}\left(\left|\delta_{h} u\right|^{\frac{q-1}{2}} \delta_{h} u\right)=\delta_{\xi}\left(\eta\left|\delta_{h} u\right|^{\frac{q-1}{2}} \delta_{h} u\right)-\left(\left|\delta_{h} u\right|^{\frac{q-1}{2}} \delta_{h} u\right)_{\xi} \delta_{\xi} \eta
$$

We arrive at

$$
\begin{aligned}
& \left.\left\|\frac{\delta_{\xi} \delta_{h} u}{|\xi|^{\frac{2 s}{q+1}}|h|^{\frac{1+\vartheta q}{q+1}}}\right\|\right|_{L^{q+1}\left(B_{r}\right)} ^{q+1} \\
& \quad \leq C_{16}\left\|\frac{\delta \xi}{|\xi|^{s}}\left(\frac{\left|\delta_{h} u\right|^{\frac{q-1}{2}}\left(\delta_{h} u\right) \eta}{|h|^{\frac{1+\vartheta q}{2}}}\right)\right\|_{L^{2}\left(\mathbb{R}^{n}\right)}^{2}+C_{16}\left\|\frac{\delta_{\xi} \eta}{|\xi|^{s}} \frac{\left(\left|\delta_{h} u\right|^{\frac{q-1}{2}} \delta_{h} u\right)_{\xi}}{|h|^{\frac{1+\vartheta q}{2}}}\right\|_{L^{2}\left(\mathbb{R}^{n}\right)}^{2},
\end{aligned}
$$

where $C_{16}=2 C_{15}$. By applying the first part of Proposition 2.6 with

$$
\psi=\frac{\left|\delta_{h} u\right|^{\frac{q-1}{2}}\left(\delta_{h} u\right) \eta}{|h|^{\frac{1+\vartheta q}{2}}}
$$

for the first term on the right-hand side of (24) we obtain 


$$
\begin{aligned}
\sup _{|\xi|>0}\left\|\frac{\delta \xi}{|\xi|^{s}}\left(\frac{\left|\delta_{h} u\right|^{\frac{q-1}{2}}\left(\delta_{h} u\right) \eta}{|h|^{\frac{1+\vartheta q}{2}}}\right)\right\|_{L^{2}\left(\mathbb{R}^{n}\right)}^{2} & \leq C_{17}\left(\frac{R}{r}\right)^{n}\left(\frac{R}{R-r}\right)^{3}\left[\frac{\left|\delta_{h} u\right|^{\frac{q-1}{2}} \delta_{h} u}{|h|^{\frac{1+\vartheta q}{2}} \eta}\right]_{W^{s, 2}\left(B_{R}\right)}^{2} \\
& \leq C_{18}\left[\frac{\left|\delta_{h} u\right|^{\frac{q-1}{2}} \delta_{h} u}{|h|^{\frac{1+\vartheta q}{2}} \eta}\right]_{W^{s, 2}\left(B_{R}\right)}^{2},
\end{aligned}
$$

where $C_{17}=C_{17}(n, s)>0$ and $C_{18}=C_{18}\left(n, s, h_{0}\right)>0$. By using that $\eta$ is Lipschitz and that $\xi<h_{0}$, along with the assumption that $\|u\|_{L^{\infty}\left(B_{1}\right)} \leq 1$ we estimate the second term on the right-hand side of (24) as follows

$$
\begin{aligned}
\left\|\frac{\delta_{\xi} \eta}{|\xi|^{s}} \frac{\left(\left|\delta_{h} u\right|^{\frac{q-1}{2}} \delta_{h} u\right)_{\xi}}{|h|^{\frac{1+\vartheta q}{2}}}\right\|_{L^{2}\left(\mathbb{R}^{n}\right)}^{2} & \leq C_{19}\left\|\frac{\left(\left|\delta_{h} u\right|^{\frac{q-1}{2}} \delta_{h} u\right)_{\xi}}{|h|^{\frac{1+\vartheta q}{2}}}\right\|_{L^{2}\left(B_{\frac{R+r}{2}+h_{0}}\right)}^{2} \\
& \leq C_{19} \int_{B_{\frac{R+r}{2}+2 h_{0}}} \frac{\left|\delta_{h} u(x)\right|^{q+1}}{|h|^{1+\vartheta q}} d x \leq C_{19} \int_{B_{R}} \frac{\left|\delta_{h} u(x)\right|^{q}}{|h|^{1+\vartheta q}} d x,
\end{aligned}
$$

where $C_{19}=C_{19}\left(n, h_{0}\right)>0$. Therefore, we arrive at

$$
\left\|\frac{\delta_{h}^{2} u}{|\xi|^{\frac{2 s}{q+1}}|h|^{\frac{1+\vartheta q}{q+1}}} \mid\right\|_{L^{q+1}\left(B_{r}\right)}^{q+1} \leq C_{20}\left[\frac{\left|\delta_{h} u\right|^{\frac{q-1}{2}} \delta_{h} u}{|h|^{\frac{1+\vartheta q}{2}} \eta}\right]_{W^{s, 2}\left(B_{R}\right)}^{2}+C_{20} \int_{B_{R}} \frac{\left|\delta_{h} u(x)\right|^{q}}{|h|^{1+\vartheta q}} d x,
$$

where $C_{20}=C_{20}\left(n, s, q, h_{0}\right)>0$. We now choose $\xi=h$ and take the supremum over $h$ for $0<|h|<h_{0}$, so that together with (23) we obtain

$$
\sup _{0<|h|<h_{0}}\left\|\frac{\delta_{h}^{2} u}{|h|^{\frac{1+2 s+\vartheta q}{q+1}}}\right\|\left\|_{L^{q+1}\left(B_{r}\right)}^{q+1} \leq C_{21} \sup _{0<|h|<h_{0}}\right\| \frac{\delta_{h} u}{|h|^{\frac{1+\vartheta q}{q}}} \|_{L^{q}\left(B_{R}\right)}^{q},
$$

where $C_{21}=C_{21}\left(n, s, q, h_{0}, \lambda\right)>0$. Next, we use the fact that by [2, Lemma 2.6] applied with $\beta=(1+\vartheta q) / q<1$, on the right-hand side of (25) we can replace the first-order difference quotient by a corresponding second-order difference quotient in the following way

$$
\sup _{0<|h|<h_{0}}\left\|\frac{\delta_{h} u}{|h|^{\frac{1+\vartheta q}{q}}}\right\| \|_{L^{q}\left(B_{R}\right)}^{q} \leq C_{22}\left(\sup _{0<|h|<h_{0}}\left\|\frac{\delta_{h}^{2} u}{|h|^{\frac{1+\vartheta q}{q}}}\right\|_{L^{q}\left(B_{R+h_{0}}\right)}^{q}+\|u\|_{L^{q}\left(B_{R+h_{0}}\right)}^{q}\right),
$$

where $C_{22}=C_{22}\left(n, q, \vartheta, h_{0}\right)>0$. By combining the last display with (25) and using that $\|u\|_{L^{q}\left(B_{R+h_{0}}\right)}^{q} \leq\|u\|_{L^{\infty}\left(B_{1}\right)}^{q}\left|B_{1}\right| \leq\left|B_{1}\right|$, we conclude that

$$
\sup _{0<|h|<h_{0}}\left\|\frac{\delta_{h}^{2} u}{|h|^{\frac{1+2 s+\vartheta q}{q+1}}}\right\|_{L^{q+1}\left(B_{r}\right)}^{q+1} \leq C\left(\sup _{0<|h|<h_{0}}\left\|\frac{\delta_{h}^{2} u}{|h|^{\frac{1+\vartheta q}{q}}}\right\|_{L^{q}\left(B_{R+4 h_{0}}\right)}^{q}+1\right),
$$

where $C=C\left(n, s, q, \vartheta, h_{0}, \lambda\right)>0$. Since $r=R-4 h_{0}$, the proof is finished.

\subsection{An iteration argument}

We now use an iteration argument based on Proposition 3.1 in order to obtain the following higher Hölder regularity result. 
Theorem 3.2 Let $R>0, x_{0} \in \mathbb{R}^{n}$ and $\lambda \geq 1$. Consider a kernel coefficient $A \in$ $\mathcal{L}_{1}\left(\lambda, B_{R}\left(x_{0}\right)\right)$, suppose that $\Phi$ satisfies (4) and (5) with respect to $\lambda$ and assume that $u \in W^{s, 2}\left(B_{R}\left(x_{0}\right)\right) \cap L_{2 s}^{1}\left(\mathbb{R}^{n}\right) \cap L^{\infty}\left(B_{R}\left(x_{0}\right)\right)$ is a local weak solution of the equation $L_{A}^{\Phi} u=0$ in $B_{R}\left(x_{0}\right)$. Then for any $0<\alpha<\min \{2 s, 1\}$, we have

$$
\begin{aligned}
{[u]_{C^{\alpha}\left(B_{R / 2}\left(x_{0}\right)\right) \leq \frac{C}{R^{\alpha}}} } & \left(\|u\|_{L^{\infty}\left(B_{R}\left(x_{0}\right)\right)}+R^{s-\frac{n}{2}}[u]_{W^{s, 2}\left(B_{R}\left(x_{0}\right)\right)}\right. \\
& \left.+R^{2 s} \int_{\mathbb{R}^{n} \backslash B_{R}\left(x_{0}\right)} \frac{|u(y)|}{\left|x_{0}-y\right|^{n+2 s}} d y\right)
\end{aligned}
$$

where $C=C(n, s, \lambda, \alpha)>0$.

Proof If $u \equiv 0$ a.e., then the assertion is trivially satisfied. Otherwise, set

$$
M_{R, x_{0}}:=\|u\|_{L^{\infty}\left(B_{R}\left(x_{0}\right)\right)}+R^{s-\frac{n}{2}}[u]_{W^{s, 2}\left(B_{R}\left(x_{0}\right)\right)}+R^{2 s} \int_{\mathbb{R}^{n} \backslash B_{R}\left(x_{0}\right)} \frac{|u(y)|}{\left|x_{0}-y\right|^{n+2 s}} d y>0 .
$$

Consider the scaled function

$$
u_{1}(x):=\frac{1}{M_{R, x_{0}}} u\left(R x+x_{0}\right)
$$

and also

$$
A_{1}(x, y):=A\left(R x+x_{0}, R y+x_{0}\right), \quad \Phi_{1}(t):=\frac{1}{M_{R, x_{0}}} \Phi\left(M_{R, x_{0}} t\right) .
$$

Observe that $u_{1}$ belongs to $W^{s, 2}\left(B_{1}\right) \cap L_{2 s}^{1}\left(\mathbb{R}^{n}\right) \cap L^{\infty}\left(B_{1}\right)$ and is a weak solution of $L_{A_{1}}^{\Phi_{1}} u_{1}=$ 0 in $B_{1}$. Moreover, it is easy to verify that $A_{1} \in \mathcal{L}_{1}\left(\lambda, B_{1}\right)$ and that $\Phi_{1}$ satisfies (4) and (5) with respect to $\lambda$. Furthermore, by using changes of variables it is straightforward to verify that $u_{1}$ satisfies

$$
\left\|u_{1}\right\|_{L^{\infty}\left(B_{1}\right)} \leq 1, \quad \int_{\mathbb{R}^{n} \backslash B_{1}} \frac{\left|u_{1}(y)\right|}{|y|^{n+2 s}} d y \leq 1, \quad\left[u_{1}\right]_{W^{s, 2}\left(B_{1}\right)} \leq 1 .
$$

Therefore, the conclusion of Proposition 3.1 is valid with respect to $u_{1}$. For $i \in \mathbb{N}_{0}$, we define the sequences

$$
q_{i}:=2+i, \quad \vartheta_{i}:=\frac{2 s i+2 s-1}{2+i} .
$$

In particular, we have

$$
\lim _{i \rightarrow \infty} q_{i}=\infty, \quad \lim _{i \rightarrow \infty} \vartheta_{i}=2 s .
$$

We split the further proof into two cases.

Case 1: $s \leq 1 / 2$. Fix $0<\alpha<2 s$. In view of (28), we can find some large enough $i_{\infty} \in \mathbb{N}$ such that

$$
\alpha<\frac{1}{q_{i_{\infty}}}+\vartheta_{i_{\infty}}-\frac{n}{q_{i_{\infty}}} .
$$

For $i=0, \ldots, i_{\infty}$, define

$$
h_{0}:=\frac{1}{64 i_{\infty}}, \quad R_{i}:=\frac{7}{8}-4(2 i+1) h_{0}=\frac{7}{8}-\frac{2 i+1}{16 i_{\infty}} .
$$

We note that

$$
R_{0}+4 h_{0}=\frac{7}{8}, \quad R_{i_{\infty}-1}-4 h_{0}=\frac{3}{4}, \quad R_{i}-4 h_{0}=R_{i+1}+4 h_{0}\left(i=0, \ldots, i_{\infty}-2\right) .
$$


Since $s \leq 1 / 2$, for $i=0, \ldots, i_{\infty}-1$ we have $0<\left(1+\vartheta_{i} q_{i}\right) / q_{i}<1$. Therefore, for $i=0, \ldots, i_{\infty}-1$ we can apply Proposition 3.1 to

$$
R=R_{i}, \quad \vartheta=\vartheta_{i}, \quad q=q_{i},
$$

so that along with (30) and the observation that by construction

$$
\frac{1+2 s+\vartheta_{i} q_{i}}{q_{i}+1}=\frac{1+\vartheta_{i+1} q_{i+1}}{q_{i+1}},
$$

we obtain the following estimates

$$
\begin{aligned}
& \left.\sup _{0<|h|<h_{0}}\left\|\frac{\delta_{h}^{2} u_{1}}{|h|^{\frac{1+\vartheta_{1} q_{1}}{q_{1}}}}\right\|\right|_{L^{q_{1}\left(B_{R_{1}+4 h_{0}}\right)}} \leq C_{0}\left(\sup _{0<|h|<h_{0}}\left\|\frac{\delta_{h}^{2} u_{1}}{|h|^{s}}\right\|_{L^{2}\left(B_{7 / 8}\right)}+1\right), \\
& \left.\sup _{0<|h|<h_{0}}\left\|\frac{\delta_{h}^{2} u_{1}}{|h|^{\frac{1+\vartheta_{i+1} q_{i+1}}{q_{i+1}}}}\right\|_{L^{q_{i+1}\left(B_{R_{i+1}+4 h_{0}}\right)}}+1\right), i=1, \ldots, i_{\infty}-2, \\
& \leq C_{0}\left(\sup _{0<|h|<h_{0}}\left\|\frac{\delta_{h}^{2} u_{1}}{|h|^{\frac{1+\vartheta_{i} q_{i}}{q_{i}}}}\right\|_{L^{q_{i}\left(B_{R_{i}+4 h_{0}}\right)}}+1\right)
\end{aligned}
$$

and

$$
\sup _{0<|h|<h_{0}}\left\|\frac{\delta_{h}^{2} u_{1}}{|h|^{\frac{1}{q_{\infty}}+\vartheta_{i \infty}}}\right\| \|_{L^{q_{i \infty}\left(B_{3 / 4}\right)}} \leq C_{0}\left(\sup _{0<|h|<h_{0}}\left\|\frac{\delta_{h}^{2} u_{1}}{|h| \frac{1+\vartheta_{i \infty}-1 q_{i \infty}-1}{q_{i_{\infty}-1}}}\right\|_{L^{q_{i \infty}-1}\left(B_{R_{i \infty}-1}+4 h_{0}\right)}+1\right),
$$

where $C_{0}=C_{0}(n, s, \lambda, \alpha)$. Combining the above estimates leads to the estimate

$$
\left.\sup _{0<|h|<h_{0}}\left\|\frac{\delta_{h}^{2} u_{1}}{|h|^{\frac{1}{q_{i \infty}}+\vartheta_{i \infty}}}\right\|\right|_{L^{q_{i \infty}\left(B_{3 / 4}\right)}} \leq C_{1}\left(\sup _{0<|h|<h_{0}}\left\|\frac{\delta_{h}^{2} u_{1}}{|h|^{s}}\right\|_{L^{2}\left(B_{7 / 8}\right)}+1\right)
$$

where $C_{1}=C_{1}(n, s, \lambda, \alpha)>0$. By taking into account the relation

$$
\delta_{h} u_{1}=\frac{1}{2}\left(\delta_{2 h} u_{1}-\delta_{h}^{2} u_{1}\right)
$$

and then using the second part of Proposition 2.6 and then (27), we deduce

$$
\begin{aligned}
& \sup _{0<|h|<h_{0}}\left\|\frac{\delta_{h}^{2} u_{1}}{|h|^{s}}\right\|_{L^{2}\left(B_{7 / 8}\right)} \leq 2 \sup _{0<|h|<h_{0}}\left\|\frac{\delta_{h} u_{1}}{|h|^{s}}\right\|_{L^{2}\left(B_{7 / 8}\right)} \\
& \leq C_{2}\left(\left[u_{1}\right]_{W^{s, 2}\left(B_{7 / 8+2 h_{0}}\right)}+\left[u_{1}\right]_{L^{\infty}\left(B_{7 / 8+2 h_{0}}\right)}\right) \\
& \leq C_{2}\left(\left[u_{1}\right]_{W^{s, 2}\left(B_{1}\right)}+\left[u_{1}\right]_{L^{\infty}\left(B_{1}\right)}\right) \leq C_{2}(n, s, \alpha) \text {. }
\end{aligned}
$$

By combining (31) with (32) and setting

$$
\beta:=\frac{1}{q_{i_{\infty}}}+\vartheta_{i_{\infty}} \in(0,1),
$$


we arrive at

$$
\sup _{0<|h|<h_{0}}\left\|\frac{\delta_{h}^{2} u_{1}}{|h|^{\beta}}\right\|_{L^{q_{i \infty}\left(B_{3 / 4}\right)}} \leq C_{3}(n, s, \lambda, \alpha) .
$$

In order to proceed, we fix a cutoff function $\chi \in C_{0}^{\infty}\left(B_{5 / 8}\right)$ with the properties

$$
0 \leq \chi \leq 1, \quad \chi \equiv 1 \text { in } B_{1 / 2}, \quad|\nabla \chi| \leq C_{4}, \quad\left|\nabla^{2} \chi\right| \leq C_{4},
$$

where by $\nabla^{2} \chi$ we denote the Hessian of $\chi$ and $C_{4}=C_{4}(n)>0$. In particular, since $0<\beta<1$, for any $h \in \mathbb{R}^{n}$ with $|h|>0$ we have

$$
\frac{\left|\delta_{h} \chi\right|}{|h|^{\beta}} \leq C_{5}, \quad \frac{\left|\delta_{h}^{2} \chi\right|}{|h|^{\beta}} \leq C_{5},
$$

where $C_{5}=C_{5}(n)>0$. Together with the identity

$$
\left.\delta_{h}^{2}\left(u_{1} \chi\right)\right)=\chi_{2 h} \delta_{h}^{2} u_{1}+2 \delta_{h} u_{1} \delta_{h} \chi_{h}+u_{1} \delta_{h}^{2} \chi,
$$

(33) and (27), for $0<|h|<h_{0}$ we obtain

$$
\begin{aligned}
& \left\|\frac{\delta_{h}^{2}\left(u_{1} \chi\right)}{|h|^{\beta}}\right\| \|_{L^{q_{i_{\infty}\left(\mathbb{R}^{n}\right)}}} \\
& \leq 2\left(\left\|\frac{\chi_{2 h} \delta_{h}^{2} u_{1}}{|h|^{\beta}}\right\|_{L^{q_{i \infty}\left(\mathbb{R}^{n}\right)}}+\left\|\frac{\delta_{h} u_{1} \delta_{h} \chi_{h}}{|h|^{\beta}}\right\|_{L^{q_{i \infty}\left(\mathbb{R}^{n}\right)}}+\left\|\frac{u_{1} \delta_{h}^{2} \chi}{|h|^{\beta}}\right\|_{L^{q_{i_{\infty}\left(\mathbb{R}^{n}\right)}}}\right)
\end{aligned}
$$

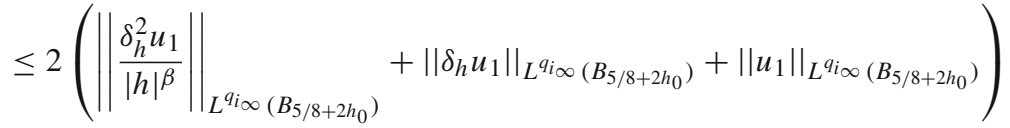

$$
\begin{aligned}
& \leq C_{6}\left(\left\|\frac{\delta_{h}^{2} u_{1}}{|h|^{\beta}}\right\|_{L^{q_{i \infty}\left(B_{3 / 4}\right)}}+\left\|u_{1}\right\|_{L^{\infty}\left(B_{3 / 4}\right)}\right) \leq C_{7}(n, s, \lambda, \alpha) .
\end{aligned}
$$

Since moreover by (27), for $|h| \geq h_{0}$ we have

$$
\left\|\frac{\delta_{h}^{2}\left(u_{1} \chi\right)}{|h|^{\beta}}\right\|_{L^{q_{i \infty}\left(\mathbb{R}^{n}\right)}} \leq C_{8}\left\|u_{1}\right\|_{L^{\infty}\left(B_{3 / 4}\right)} \leq C_{8}(n, s, \alpha) .
$$

by Lemma 2.4 it follows that

$$
\left[u_{1} \chi\right]_{\mathcal{N}_{\infty}^{\beta, q_{i \infty}\left(\mathbb{R}^{n}\right)}} \leq C_{9}\left[u_{1} \chi\right]_{\mathcal{B}_{\infty}^{\beta, q_{i \infty}\left(\mathbb{R}^{n}\right)}}=C_{9} \sup _{h>0}\left\|\frac{\delta_{h}^{2}\left(u_{1} \chi\right)}{|h|^{\beta}}\right\|_{L^{q_{i \infty}\left(\mathbb{R}^{n}\right)}} \leq C_{10}(n, s, \lambda, \alpha) .
$$

Along with Lemma 2.5 with our choice of $\beta$ and $q=q_{i_{\infty}}$ (which is applicable in view of (29)), we obtain

$$
\begin{aligned}
{\left[u_{1}\right]_{C^{\alpha}\left(B_{1 / 2}\right)}=\left[u_{1} \chi\right]_{C^{\alpha}\left(B_{1 / 2}\right)} } & \leq C_{11}\left(\left[u_{1} \chi\right]_{\mathcal{N}_{\infty}^{\beta, q_{i \infty}}\left(\mathbb{R}^{n}\right)}\right)^{\frac{\alpha q_{i \infty}+n}{\beta q_{i \infty}}}\left(\left\|u_{1} \chi\right\|_{L^{q_{i \infty}\left(\mathbb{R}^{n}\right)}}\right)^{1-\frac{\alpha q_{i_{\infty}}+n}{\beta q_{i \infty}}} \\
& \leq C_{12}\left(\left\|u_{1}\right\|_{L^{\infty}\left(B_{5 / 8}\right)}\right)^{1-\frac{\alpha q_{i \infty}+n}{\beta q_{i_{\infty}}}} \leq C(n, s, \lambda, \alpha) .
\end{aligned}
$$

Finally, rescaling yields the desired estimate, namely (26). This finishes the proof in the case when $s \leq 1 / 2$. 
Case 2: $s>1 / 2$. Fix $0<\alpha<1$. Since in view of (28) we have

$$
\lim _{i \rightarrow \infty} \frac{1+\vartheta_{i} q_{i}}{q_{i}}=2 s>1
$$

and the expression $\frac{1+\vartheta_{i} q_{i}}{q_{i}}$ is increasing in $i$, there exists some $i_{\infty} \in \mathbb{N}$ such that

$$
\frac{1+\vartheta_{i} q_{i}}{q_{i}}<1 \text { for any } i=0, \ldots, i_{\infty}-1 \text { and } \frac{1+\vartheta_{i_{\infty}} q_{i_{\infty}}}{q_{i_{\infty}}} \geq 1 .
$$

Next, we choose $j_{\infty} \in \mathbb{N}$ large enough such that

$$
\alpha<1-\frac{n}{i_{\infty}+j_{\infty}} .
$$

Moreover, we choose some $\varepsilon \in(0,1)$ such that

$$
\alpha<1-\varepsilon-\frac{n}{i_{\infty}+j_{\infty}}
$$

and let $\gamma:=1-\varepsilon$. Furthermore, similar to the previous case, for $i=0, \ldots, i_{\infty}+j_{\infty}$ we define

$$
h_{0}:=\frac{1}{64\left(i_{\infty}+j_{\infty}\right)}, \quad R_{i}:=\frac{7}{8}-4(2 i+1) h_{0}=\frac{7}{8}-\frac{2 i+1}{16\left(i_{\infty}+j_{\infty}\right)}
$$

and note that

$$
R_{0}+4 h_{0}=\frac{7}{8}, \quad R_{i_{\infty}+j_{\infty}-1}-4 h_{0}=\frac{3}{4}, \quad R_{i}-4 h_{0}=R_{i+1}+4 h_{0}\left(i=0, \ldots, i_{\infty}+j_{\infty}-2\right) .
$$

In view of (37), for $i=0, \ldots, i_{\infty}-1$ we can apply Proposition 3.1 to

$$
R=R_{i}, \quad \vartheta=\vartheta_{i}, \quad q=q_{i},
$$

which in almost exactly the same way as in Case 1 (cf. (33)) leads to the estimate

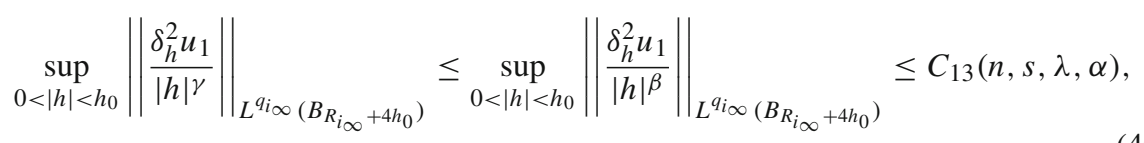

where we used that by (37) we have $\gamma<1 \leq \beta=\frac{1}{q_{i_{\infty}}}+\vartheta_{i_{\infty}}$. Next, we set $\widetilde{\vartheta}_{i}:=\gamma-\frac{1}{q_{i}}$ and observe that

$$
\frac{1+\widetilde{\vartheta}_{i} q_{i}}{q_{i}}=\gamma \in(0,1)
$$

Therefore, for $i=i_{\infty}, \ldots, i_{\infty}+j_{\infty}-1$ we can apply Proposition 3.1 to

$$
R=R_{i}, \quad \vartheta=\tilde{\vartheta}_{i}, \quad q=q_{i},
$$

so that along with (39) and the observation that $s>1 / 2$ implies

$$
\frac{1+2 s+\tilde{\vartheta}_{i} q_{i}}{q_{i}+1}>\frac{2+\tilde{\vartheta}_{i} q_{i}}{q_{i}+1}=1+\frac{q_{i}(\gamma-1)}{q_{i}+1}>\gamma,
$$

we obtain the estimates

$$
\sup _{0<|h|<h_{0}}\left\|\frac{\delta_{h}^{2} u_{1}}{|h|^{\gamma}}\right\|_{L^{q_{i+1}\left(B_{R_{i+1}+4 h_{0}}\right)}}
$$




$$
\leq C_{14}\left(\sup _{0<|h|<h_{0}}\left\|\frac{\delta_{h}^{2} u_{1}}{|h|^{\gamma}}\right\|_{L^{q_{i}\left(B_{R_{i}+4 h_{0}}\right)}}+1\right), i=i_{\infty}, \ldots, i_{\infty}+j_{\infty}-1
$$

and

$$
\sup _{0<|h|<h_{0}}\left\|\frac{\delta_{h}^{2} u_{1}}{|h|^{\gamma}}\right\|_{L^{q_{\infty}+j \infty}\left(B_{3 / 4}\right)} \leq C_{14}\left(\sup _{0<|h|<h_{0}}\left\|\frac{\delta_{h}^{2} u_{1}}{|h|^{\gamma}}\right\|_{L^{q_{i_{\infty}+j_{\infty}-1}\left(B_{R_{i_{\infty}+j \infty-1}+4 h_{0}}\right)}}+1\right),
$$

where $C_{14}=C_{14}(n, s, \lambda, \alpha)$. Combining these estimates with (40) and recalling that $\gamma=$ $1-\varepsilon$, we arrive at

$$
\sup _{0<|h|<h_{0}}\left\|\frac{\delta_{h}^{2} u_{1}}{|h|^{1-\varepsilon}}\right\|_{L^{q_{i \infty+j \infty(B / 4)}}} \leq C_{15}(n, s, \lambda, \alpha) .
$$

By imitating the arguments used to conclude in case 1 (cf. (35) and (36)), which in particular involves applying Lemma 2.5 with $\beta=1-\varepsilon$ and $q=q_{i_{\infty}+j_{\infty}}$ (which is applicable in view of (38)), we conclude that

$$
\left[u_{1}\right]_{C^{\alpha}\left(B_{1 / 2}\right)} \leq C=C(n, s, \lambda, \alpha)
$$

for a different constant $C$ as the one in (36). The desired estimate (26) now once again simply follows by rescaling, which finishes the proof.

\section{Higher Hölder regularity by approximation}

We now use an approximation argument inspired by [2, section 6] and [5] in order to prove Theorem 1.1 and Theorem 1.2 under full generality. In order to do so, we need the following definition.

Definition Let $0<r<R$ and let $u \in W^{s, 2}\left(B_{R}\right) \cap L_{2 s}^{1}\left(\mathbb{R}^{n}\right)$. We say that $v \in W^{s, 2}\left(B_{R}\right) \cap$ $L_{2 s}^{1}\left(\mathbb{R}^{n}\right)$ is a weak solution of the problem

$$
\begin{cases}L_{A}^{\Phi} v=0 & \text { in } B_{r} \\ v=u & \text { a.e. in } \mathbb{R}^{n} \backslash B_{r},\end{cases}
$$

if we have $\mathcal{E}_{A}^{\Phi}(u, \varphi)=0$ for any $\varphi \in W_{0}^{s, 2}\left(B_{r}\right)$ and $v=u$ a.e. in $\mathbb{R}^{n} \backslash B_{r}$.

Lemma 4.1 Let $s \in(0,1), \lambda \geq 1, q>\frac{n}{2 s}$ and $M \geq 1$. Then for any $\tau>0$, there exists some small enough $\delta=\delta(\tau, n, s, \lambda, q, M)>0$ such that the following is true. Assume that $\Phi$ satisfies (4) and (5) with respect to $\lambda$, that $A \in \mathcal{L}_{0}(\lambda)$ and that we have $f \in L^{q}\left(B_{1}\right)$. Moreover, suppose that $\widetilde{A}$ is another kernel coefficient of class $\mathcal{L}_{0}(\lambda)$ such that

$$
\|A-\widetilde{A}\|_{L^{\infty}\left(\mathbb{R}^{n} \times \mathbb{R}^{n}\right)} \leq \delta, \quad\|f\|_{L^{q}\left(B_{1}\right)} \leq \delta,
$$

and let $u \in W^{s, 2}\left(B_{1}\right) \cap L_{2 s}^{1}\left(\mathbb{R}^{n}\right)$ be a local weak solution of

$$
L_{A}^{\Phi} u=f \text { in } B_{1}
$$

that satisfies

$$
\sup _{x \in B_{1}}|u(x)|+\int_{\mathbb{R}^{n} \backslash B_{1}} \frac{|u(y)|}{|y|^{n+2 s}} d y \leq M
$$


Then the unique weak solution $v \in W^{s, 2}\left(B_{1}\right) \cap L_{2 s}^{1}\left(\mathbb{R}^{n}\right)$ of the problem

$$
\begin{cases}L_{\widetilde{A}}^{\Phi} v=0 & \text { in } B_{7 / 8} \\ v=u & \text { a.e. in } \mathbb{R}^{n} \backslash B_{7 / 8}\end{cases}
$$

satisfies

$$
\|u-v\|_{L^{\infty}\left(B_{3 / 4}\right)} \leq \tau .
$$

Proof First of all, we remark that the existence of a unique weak solution of the problem (44) belonging to $W^{s, 2}\left(B_{1}\right) \cap L_{2 s}^{1}\left(\mathbb{R}^{n}\right)$ can be shown almost exactly as in [16, Theroem 1 and Remark 3] by using the theory of monotone operators and additionally taking into account the bounds (4) and (5) imposed on $\Phi$.

We now prove by contradiction. Assume that the conclusion is not true. Then there exist some $\tau>0$, sequences of kernel coefficients $\left\{A_{m}\right\}_{m=1}^{\infty}$ and $\left\{\widetilde{A}_{m}\right\}_{m=1}^{\infty}$ of class $\mathcal{L}_{0}(\lambda)$, a sequence of functions $\left\{\Phi_{m}\right\}_{m=1}^{\infty}$ satisfying (4) and (5), and sequences $\left\{u_{m}\right\}_{k=1}^{\infty} \subset W^{s, 2}\left(B_{1}\right) \cap L_{2 s}^{1}\left(\mathbb{R}^{n}\right)$, $\left\{f_{m}\right\}_{m=1}^{\infty} \subset L^{q}\left(B_{1}\right)$, such that for any $m$ the function $u_{m}$ is a local weak solution of the problem

$$
\begin{aligned}
& L_{A_{m}}^{\Phi_{m}} u_{m}=f_{m} \text { in } B_{1}, \\
& \sup _{x \in B_{1}}\left|u_{m}(x)\right|+\int_{\mathbb{R}^{n} \backslash B_{1}} \frac{\left|u_{m}(y)\right|}{|y|^{n+2 s}} d y \leq M, \\
& \left\|A_{m}-\widetilde{A}_{m}\right\|_{L^{\infty}\left(\mathbb{R}^{n} \times \mathbb{R}^{n}\right)} \leq \frac{1}{m}, \quad\left\|f_{m}\right\|_{L^{q}\left(B_{1}\right)} \leq \frac{1}{m},
\end{aligned}
$$

but for any $m$ the unique weak solution $v_{m} \in W^{s, 2}\left(B_{1}\right) \cap L_{2 s}^{1}\left(\mathbb{R}^{n}\right)$ of

$$
\begin{cases}L_{\widetilde{A}_{m}}^{\Phi_{m}} v_{m}=0 & \text { in } B_{7 / 8} \\ v_{m}=u_{m} & \text { a.e. in } \mathbb{R}^{n} \backslash B_{7 / 8}\end{cases}
$$

satisfies

$$
\left\|u_{m}-v_{m}\right\|_{L^{\infty}\left(B_{3 / 4}\right)}>\tau \text {. }
$$

In view of (2), (5) and using $w_{m}:=u_{m}-v_{m} \in W_{0}^{s, 2}\left(B_{7 / 8}\right)$ as a test function in (49) and also in (46), we obtain

$$
\begin{aligned}
& \int_{\mathbb{R}^{n}} \int_{\mathbb{R}^{n}} \frac{\left(w_{m}(x)-w_{m}(y)\right)^{2}}{|x-y|^{n+2 s}} d y d x \\
& \leq \lambda \int_{\mathbb{R}^{n}} \int_{\mathbb{R}^{n}} \widetilde{A}_{m}(x, y) \frac{\left(\left(u_{m}(x)-u_{m}(y)\right)-\left(v_{m}(x)-v_{m}(y)\right)\right)^{2}}{|x-y|^{n+2 s}} d y d x \\
& \leq \lambda^{2}\left(\int_{\mathbb{R}^{n}} \int_{\mathbb{R}^{n}} \widetilde{A}_{m}(x, y) \frac{\Phi_{m}\left(u_{m}(x)-u_{m}(y)\right)\left(w_{m}(x)-w_{m}(y)\right)}{|x-y|^{n+2 s}} d y d x\right. \\
& -\underbrace{\int_{\mathbb{R}^{n}} \int_{\mathbb{R}^{n}} \widetilde{A}_{m}(x, y) \frac{\Phi_{m}\left(v_{m}(x)-v_{m}(y)\right)\left(w_{m}(x)-w_{m}(y)\right)}{|x-y|^{n+2 s}} d y d x}_{=0}) \\
& =\lambda^{2}\left(\int_{\mathbb{R}^{n}} \int_{\mathbb{R}^{n}}\left(\widetilde{A}_{m}(x, y)-A_{m}(x, y)\right) \frac{\Phi_{m}\left(u_{m}(x)-u_{m}(y)\right)\left(w_{m}(x)-w_{m}(y)\right)}{|x-y|^{n+2 s}} d y d x\right. \\
& \left.+\int_{\mathbb{R}^{n}} \int_{\mathbb{R}^{n}} A_{m}(x, y) \frac{\Phi_{m}\left(u_{m}(x)-u_{m}(y)\right)\left(w_{m}(x)-w_{m}(y)\right)}{|x-y|^{n+2 s}} d y d x\right)
\end{aligned}
$$




$$
\begin{aligned}
= & \underbrace{\lambda^{2} \int_{\mathbb{R}^{n}} \int_{\mathbb{R}^{n}}\left(\widetilde{A}_{m}(x, y)-A_{m}(x, y)\right) \frac{\Phi_{m}\left(u_{m}(x)-u_{m}(y)\right)\left(w_{m}(x)-w_{m}(y)\right)}{|x-y|^{n+2 s}} d y d x}_{=: I_{1}} \\
& +\underbrace{\lambda^{2} \int_{B_{1}} f_{m}(x) w_{m}(x) d x}_{:=I_{2}} .
\end{aligned}
$$

By using (4) and (48), we further estimate $I_{1}$ as follows

$$
\begin{aligned}
& I_{1} \leq \lambda^{3} \int_{\mathbb{R}^{n}} \int_{\mathbb{R}^{n}}\left|\tilde{A}_{m}(x, y)-A_{m}(x, y)\right| \frac{\left|u_{m}(x)-u_{m}(y)\right|\left|w_{m}(x)-w_{m}(y)\right|}{|x-y|^{n+2 s}} d y d x \\
& \leq \lambda^{3}\left\|A_{m}-\widetilde{A}_{m}\right\|_{L^{\infty}\left(\mathbb{R}^{n} \times \mathbb{R}^{n}\right)} \int_{\mathbb{R}^{n}} \int_{\mathbb{R}^{n}} \frac{\left|u_{m}(x)-u_{m}(y)\right|\left|w_{m}(x)-w_{m}(y)\right|}{|x-y|^{n+2 s}} d y d x \\
& \leq \lambda^{3} \frac{1}{m} \underbrace{\int_{B_{15 / 16}} \int_{B_{15 / 16}} \frac{\left|u_{m}(x)-u_{m}(y)\right|\left|w_{m}(x)-w_{m}(y)\right|}{|x-y|^{n+2 s}} d y d x}_{=: I_{1,1}} \\
& +2 \lambda^{3} \frac{1}{m} \underbrace{\int_{B_{7 / 8}} \int_{\mathbb{R}^{n} \backslash B_{15 / 16}} \frac{\left|u_{m}(x)\right|\left|w_{m}(x)\right|}{|x-y|^{n+2 s}} d y d x}_{=: I_{1,2}} \\
& +2 \lambda^{3} \frac{1}{m} \underbrace{\int_{B_{7 / 8}} \int_{\mathbb{R}^{n} \backslash B_{15 / 16}} \frac{\left|u_{m}(y)\right|\left|w_{m}(x)\right|}{|x-y|^{n+2 s}} d y d x}_{=: I_{1,3}} .
\end{aligned}
$$

In order to proceed, we observe that since $n>2 s$, we have $q>\frac{n}{2 s}>\frac{2 n}{n+2 s}$, so that Hölder's inequality and (48) yield

$$
\left(\int_{B_{1}}\left|f_{m}(x)\right|^{\frac{2 n}{n+2 s}} d x\right)^{\frac{n+2 s}{2 n}} \leq C_{1}\left\|f_{m}\right\|_{L^{q}\left(B_{1}\right)} \leq \frac{C_{1}}{m},
$$

where $C_{1}=C_{1}(n, s, q)>0$. By using the Cauchy-Schwarz inequality, Theorem 2.10, (47) and (51), for $I_{1,1}$ we obtain

$$
\begin{aligned}
I_{1,1} \leq & \left(\int_{B_{15 / 16}} \int_{B_{15 / 16}} \frac{\left(u_{m}(x)-u_{m}(y)\right)^{2}}{|x-y|^{n+2 s}} d y d x\right)^{\frac{1}{2}}\left(\int_{\mathbb{R}^{n}} \int_{\mathbb{R}^{n}} \frac{\left(w_{m}(x)-w_{m}(y)\right)^{2}}{|x-y|^{n+2 s}} d y d x\right)^{\frac{1}{2}} \\
\leq & C_{2}\left(\left\|u_{m}\right\|_{L^{2}\left(B_{1}\right)}^{2}+|| u_{m} \|_{L^{1}\left(B_{1}\right)} \int_{\mathbb{R}^{n} \backslash B_{1}} \frac{\left|u_{m}(y)\right|}{|y|^{n+2 s}} d y+\left(\int_{B_{1}}\left|f_{m}(x)\right|^{\frac{2 n}{n+2 s}} d x\right)^{\frac{n+2 s}{n}}\right)^{\frac{1}{2}} \\
& \times\left(\int_{\mathbb{R}^{n}} \int_{\mathbb{R}^{n}} \frac{\left(w_{m}(x)-w_{m}(y)\right)^{2}}{|x-y|^{n+2 s}} d y d x\right)^{\frac{1}{2}} \\
\leq & C_{3}\left(\int_{\mathbb{R}^{n}} \int_{\mathbb{R}^{n}} \frac{\left(w_{m}(x)-w_{m}(y)\right)^{2}}{|x-y|^{n+2 s}} d y d x\right)^{\frac{1}{2}},
\end{aligned}
$$

where $C_{2}$ and $C_{3}$ depend only on $n, s, \lambda, q$ and $M$. For $I_{1,2}$, by using Lemma 2.1, the CauchySchwarz-inequality, the fractional Friedrichs-Poincaré inequality (Lemma 2.3) and (47), we 
have

$$
\begin{aligned}
I_{1,2} & \leq C_{4} \int_{B_{7 / 8}} \int_{\mathbb{R}^{n} \backslash B_{15 / 16}} \frac{\left|u_{m}(x)\right|\left|w_{m}(x)\right|}{|y|^{n+2 s}} d y d x \\
& =C_{5} \int_{B_{7 / 8}}\left|u_{m}(x) \| w_{m}(x)\right| d x \\
& \leq C_{5}|| w_{m}\left\|_{L^{2}\left(B_{7 / 8}\right)}|| u_{m}\right\|_{L^{2}\left(B_{7 / 8}\right)} \leq C_{6}\left(\int_{\mathbb{R}^{n}} \int_{\mathbb{R}^{n}} \frac{\left(w_{m}(x)-w_{m}(y)\right)^{2}}{|x-y|^{n+2 s}} d y d x\right)^{\frac{1}{2}},
\end{aligned}
$$

where $C_{4}=15^{n+2 s}, C_{5}=C_{5}(n, s)>0$ and $C_{6}=C_{6}(n, s, M)>0$. Similarly, by using Lemma 2.1, the Cauchy-Schwarz-inequality, Lemma 2.2, Lemma 2.3 and (47), for $I_{1,3}$ we obtain

$$
\begin{aligned}
I_{1,3} & \leq C_{4} \int_{B_{7 / 8}} \int_{\mathbb{R}^{n} \backslash B_{15 / 16}} \frac{\left|u_{m}(y)\right|\left|w_{m}(x)\right|}{|y|^{n+2 s}} d y d x \\
& \leq C_{7}|| w_{m} \|_{L^{2}\left(B_{7 / 8}\right)} \int_{\mathbb{R}^{n} \backslash B_{15 / 16}} \frac{\left|u_{m}(y)\right|}{|y|^{n+2 s}} d y \\
& \leq C_{8}\left(\int_{\mathbb{R}^{n}} \int_{\mathbb{R}^{n}} \frac{\left(w_{m}(x)-w_{m}(y)\right)^{2}}{|x-y|^{n+2 s}} d y d x\right)^{\frac{1}{2}}\left(|| u_{m} \|_{L^{1}\left(B_{1}\right)}+\int_{\mathbb{R}^{n} \backslash B_{1}} \frac{\left|u_{m}(y)\right|}{|y|^{n+2 s}} d y\right) \\
& \leq C_{9}\left(\int_{\mathbb{R}^{n}} \int_{\mathbb{R}^{n}} \frac{\left(w_{m}(x)-w_{m}(y)\right)^{2}}{|x-y|^{n+2 s}} d y d x\right)^{\frac{1}{2}},
\end{aligned}
$$

where again all the constants depend only on $n, s$ and $M$. Next, by using Hölder's inequality, the fractional Sobolev inequality (cf. [9, Theorem 6.5]) and (51), we estimate $I_{2}$ in the following way

$$
\begin{aligned}
I_{2} & \leq\left(\int_{B_{1}}\left|f_{m}(x)\right|^{\frac{2 n}{n+2 s}} d x\right)^{\frac{n+2 s}{2 n}}\left(\int_{B_{1}}\left|w_{m}(x)\right|^{\frac{2 n}{n-2 s}} d x\right)^{\frac{n-2 s}{2 n}} \\
& \leq C_{10} \frac{1}{m}\left(\int_{\mathbb{R}^{n}} \int_{\mathbb{R}^{n}} \frac{\left(w_{m}(x)-w_{m}(y)\right)^{2}}{|x-y|^{n+2 s}} d y d x\right)^{\frac{1}{2}},
\end{aligned}
$$

where $C_{10}=C_{10}(n, s, q)>0$. Putting the above estimates together, we arrive at

$$
\left(\int_{\mathbb{R}^{n}} \int_{\mathbb{R}^{n}} \frac{\left(w_{m}(x)-w_{m}(y)\right)^{2}}{|x-y|^{n+2 s}} d y d x\right)^{\frac{1}{2}} \leq \frac{C_{11}}{m}
$$

for some $C_{11}=C_{11}(n, s, \lambda, q, M)>0$. Combining this estimate with the fractional Friedrichs-Poincaré inequality (Lemma 2.3) leads to

$$
\left\|w_{m}\right\|_{L^{2}\left(B_{7 / 8}\right)} \leq C_{12}\left(\int_{\mathbb{R}^{n}} \int_{\mathbb{R}^{n}} \frac{\left(w_{m}(x)-w_{m}(y)\right)^{2}}{|x-y|^{n+2 s}} d y d x\right)^{\frac{1}{2}} \leq C_{12} \frac{C_{11}}{m} \stackrel{m \rightarrow \infty}{\longrightarrow} 0 .
$$

In other words, we have

$$
\lim _{m \rightarrow \infty}\left\|u_{m}-v_{m}\right\|_{L^{2}\left(B_{7 / 8}\right)}=0
$$


In view of Theorem 2.11, Theorem 2.12, the fact that $u_{m}=v_{m}$ a.e. in $\mathbb{R}^{n} \backslash B_{7 / 8}$ and Lemma 2.2, we have

$$
\begin{aligned}
\sup _{x \in \bar{B}_{3 / 4}}\left|v_{m}(x)\right|+\left[v_{m}\right]_{C^{\beta}\left(B_{3 / 4}\right)} & \leq C_{13}\left(\left\|v_{m}\right\|_{L^{2}\left(B_{7 / 8}\right)}+\int_{\mathbb{R}^{n} \backslash B_{7 / 8}} \frac{\left|v_{m}(y)\right|}{|y|^{n+2 s}} d y\right) \\
& \leq C_{13}\left(\left\|w_{m}\right\|_{L^{2}\left(B_{7 / 8}\right)}+\left\|u_{m}\right\|_{L^{2}\left(B_{7 / 8}\right)}+\int_{\mathbb{R}^{n} \backslash B_{7 / 8}} \frac{\left|u_{m}(y)\right|}{|y|^{n+2 s}} d y\right) \\
& \leq C_{14}\left(\left\|w_{m}\right\|_{L^{2}\left(B_{7 / 8}\right)}+\left\|u_{m}\right\|_{L^{\infty}\left(B_{1}\right)}+\int_{\mathbb{R}^{n} \backslash B_{1}} \frac{\left|u_{m}(y)\right|}{|y|^{n+2 s}} d y\right),
\end{aligned}
$$

so that in view of (52) and (47) the sequence $\left\{v_{m}\right\}_{m=1}^{\infty}$ is uniformly bounded in $\bar{B}_{3 / 4}$ and has uniformly bounded $C^{\beta}$ seminorms in $B_{3 / 4}$, where $\beta=\beta(n, s, \lambda, q)>0$. Moreover, in view of (47) and Theorem 2.12, the sequence $\left\{u_{m}\right\}_{m=1}^{\infty}$ is also uniformly bounded in $\bar{B}_{3 / 4}$ and has uniformly bounded $C^{\beta}$ seminorms in $B_{3 / 4}$. In particular, the same is also true for the sequence $\left\{u_{m}-v_{m}\right\}_{m=1}^{\infty}$. Therefore, by the Arzelà-Ascoli theorem, by passing to a subsequence if necessary, we obtain that the sequence $\left\{u_{m}-v_{m}\right\}_{m=1}^{\infty}$ converges uniformly in $\bar{B}_{3 / 4}$ to some function $h$. Since by (53) up to passing to another subsequence we have

$$
u_{m}-v_{m} \stackrel{m \rightarrow \infty}{\longrightarrow} 0 \text { a.e. in } B_{7 / 8},
$$

which by uniqueness of the limit implies that $h=0$ a.e. in $B_{3 / 4}$, we arrive at

$$
\lim _{m \rightarrow \infty}\left\|u_{m}-v_{m}\right\|_{L^{\infty}\left(B_{3 / 4}\right)}=0 .
$$

In particular, for $m$ large enough we have

$$
\left\|u_{m}-v_{m}\right\|_{L^{\infty}\left(B_{3 / 4}\right)} \leq \tau,
$$

which contradicts (50). This finishes the proof.

Next, we use the above Lemma and Theorem 3.2 in order to prove the desired higher Hölder regularity in the case when $A$ is close enough to a locally translation invariant kernel coefficient.

Proposition 4.2 Let $s \in(0,1), \lambda \geq 1, q>\frac{n}{2 s}$ and let $\Theta=\min \left\{2 s-\frac{n}{q}, 1\right\}$. Then for any $0<\varepsilon<\Theta$, there exists some small enough $\delta=\delta(\varepsilon, n, s, \lambda, q)>0$ such that the following is true. Assume that $\Phi$ satisfies (4) and (5) with respect to $\lambda$, that $A \in \mathcal{L}_{0}(\lambda)$ and that we have $f \in L^{q}\left(B_{1}\right)$. Moreover, suppose that there exists a kernel coefficient $\widetilde{A} \in \mathcal{L}_{1}\left(B_{1}, \lambda\right)$ such that

$$
\|A-\tilde{A}\|_{L^{\infty}\left(\mathbb{R}^{n} \times \mathbb{R}^{n}\right)} \leq \delta, \quad\|f\|_{L^{q}\left(B_{1}\right)} \leq \delta .
$$

Then for any local weak solution $u \in W^{s, 2}\left(B_{1}\right) \cap L_{2 s}^{1}\left(\mathbb{R}^{n}\right)$ of

$$
L_{A}^{\Phi} u=f \text { in } B_{1}
$$

that satisfies

$$
\sup _{x \in B_{1}}|u(x)| \leq 1, \quad \int_{\mathbb{R}^{n} \backslash B_{1}} \frac{|u(y)|}{|y|^{n+2 s}} d y \leq 1,
$$

we have $u \in C^{\Theta-\varepsilon}\left(\bar{B}_{1 / 2}\right)$ and

$$
[u]_{C^{\alpha}\left(B_{1 / 2}\right)} \leq C(n, s, \lambda, q) .
$$


Proof We divide the proof into two parts.

Step 1: Regularity at the origin. In this step, our aim is to prove that for any $0<\varepsilon<\Theta$ and any $0<r<1$, there exists some small enough $\delta>0$ such that if $A, \widetilde{A}, f$ and $u$ are as above, then

$$
\sup _{x \in B_{r}}|u(x)-u(0)| \leq C_{1} r^{\Theta-\varepsilon}
$$

for some constant $C_{1}=C_{1}(n, s, \lambda, \varepsilon)>0$. In order to accomplish this, we fix some $0<$ $\varepsilon<\Theta$ and observe that it suffices to prove that there exist $0<\rho<\frac{1}{3}$ and $\delta>0$ such that if $A, \widetilde{A}, f$ and $u$ are as above, then for any $k \in \mathbb{N}_{0}$ we have

$$
\sup _{x \in B_{\rho^{k}}}|u(x)-u(0)| \leq 2 \rho^{k(\Theta-\varepsilon)}, \quad \int_{\mathbb{R}^{n} \backslash B_{1}} \frac{\left|u\left(\rho^{k} y\right)-u(0)\right|}{\rho^{k(\Theta-\varepsilon)}|y|^{n+2 s}} d y \leq M_{0},
$$

where $M_{0}:=1+\int_{\mathbb{R}^{n} \backslash B_{1}} \frac{d y}{|y|^{n+2 s}}<\infty$. Indeed, assume that (59) were true. Since for any $0<r<1$ there exists some $k \in \mathbb{N}_{0}$ such that $\rho^{k+1}<r \leq \rho^{k}$, by the first inequality in (59) we would arrive at

$$
\sup _{x \in B_{r}}|u(x)-u(0)| \leq \sup _{x \in B_{\rho^{k}}}|u(x)-u(0)| \leq 2 \rho^{k(\Theta-\varepsilon)}=\frac{2}{\rho^{\Theta-\varepsilon}} \rho^{(k+1)(\Theta-\varepsilon)} \leq \frac{2}{\rho^{\Theta-\varepsilon}} r^{\Theta-\varepsilon},
$$

which would prove (58) with $C_{1}=\frac{2}{\rho^{\Theta-\varepsilon}}$.

In order to prove (59), we proceed by induction. In the case when $k=0,(59)$ is true by the assumptions (57).

Next, suppose that (59) holds up to $k$ and let us prove that it is also true for $k+1$. Let $\tau>0$ to be chosen small enough and consider the corresponding $\delta=\delta(\tau, n, s, \lambda, q, M)>0$ given by Lemma 4.1 , where $M:=2+M_{0}$. Assume that (55) is satisfied with respect to this $\delta$. Furthermore, define

$$
w_{k}(x):=\frac{u\left(\rho^{k} x\right)-u(0)}{\rho^{k(\Theta-\varepsilon)}}, \quad f_{k}(x):=\rho^{k(2 s-(\Theta-\varepsilon))} f\left(\rho^{k} x\right)
$$

and

$$
A_{k}(x, y):=A\left(\rho^{k} x, \rho^{k} y\right), \quad \widetilde{A}_{k}(x, y):=\widetilde{A}\left(\rho^{k} x, \rho^{k} y\right), \quad \Phi_{k}(t):=\frac{1}{\rho^{k(\Theta-\varepsilon)}} \Phi\left(\rho^{k(\Theta-\varepsilon)} t\right) .
$$

We note that $A_{k} \in \mathcal{L}_{0}(\lambda), \widetilde{A}_{k} \in \mathcal{L}_{1}\left(\lambda, B_{\frac{1}{\rho^{k}}}\right) \subset \mathcal{L}_{1}\left(\lambda, B_{1}\right)$ and that $\Phi_{k}$ satisfies (4) and (5) with respect to $\lambda$. Moreover, $w_{k}$ belongs to $W^{s, 2}\left(B_{1}\right) \cap L_{2 s}^{1}\left(\mathbb{R}^{n}\right)$ and is a local weak solution of $L_{A_{k}}^{\Phi_{k}} w_{k}=f_{k}$ in $B_{1}$, while by (55) we have

$$
\left\|A_{k}-\widetilde{A}_{k}\right\|_{L^{\infty}\left(\mathbb{R}^{n} \times \mathbb{R}^{n}\right)}=\|A-\widetilde{A}\|_{L^{\infty}\left(\mathbb{R}^{n} \times \mathbb{R}^{n}\right)} \leq \delta
$$

and

$$
\left\|f_{k}\right\|_{L^{q}\left(B_{1}\right)}=\rho^{k(2 s-(\Theta-\varepsilon))} \rho^{-k \frac{n}{q}}\|f\|_{L^{q}\left(B_{\rho^{k}}\right)} \leq\|f\|_{L^{q}\left(B_{1}\right)} \leq \delta,
$$

where we have also used that $\Theta \leq 2 s-\frac{n}{q}$ and thus $k\left(2 s-(\Theta-\varepsilon)-\frac{n}{q}\right) \geq k \varepsilon \geq 0$. Moreover, by the induction hypothesis we have

$$
\left\|w_{k}\right\|_{L^{\infty}\left(B_{1}\right)} \leq 2, \quad \int_{\mathbb{R}^{n} \backslash B_{1}} \frac{\left|w_{k}(y)\right|}{|y|^{n+2 s}} d y \leq M_{0} .
$$


Therefore, by Lemma 4.1 the unique weak solution $v_{k} \in W^{s, 2}\left(B_{1}\right) \cap L_{2 s}^{1}\left(\mathbb{R}^{n}\right)$ of

$$
\begin{cases}L_{\widetilde{A}_{k}}^{\Phi_{k}} v_{k}=0 & \text { in } B_{7 / 8} \\ v_{k}=w_{k} & \text { a.e. in } \mathbb{R}^{n} \backslash B_{7 / 8}\end{cases}
$$

satisfies

$$
\left\|w_{k}-v_{k}\right\|_{L^{\infty}\left(B_{3 / 4}\right)} \leq \tau .
$$

Together with the fact that $w_{k}(0)=0$, we obtain that for any $x \in B_{1 / 3}$ we have

$$
\begin{aligned}
\left|w_{k}(x)\right| & \leq\left|w_{k}(x)-v_{k}(x)\right|+\left|v_{k}(0)-w_{k}(0)\right|+\left|v_{k}(x)-v_{k}(0)\right| \\
& \leq 2 \tau+\left[v_{k}\right]_{C^{\Theta-\varepsilon / 2}\left(B_{1 / 3}\right)}|x|^{\Theta-\varepsilon / 2} .
\end{aligned}
$$

Our next goal is to prove that the right-hand side of the previous estimate is uniformly bounded by a constant that does not depend on $k$. In order to do so, we observe that since $\widetilde{A}_{k} \in \mathcal{L}_{1}\left(\lambda, B_{1}\right) \subset \mathcal{L}_{1}\left(\lambda, B_{2 / 3}\right)$, by Theorem 3.2 we have

$$
\left[v_{k}\right]_{C^{\Theta-\varepsilon / 2}\left(B_{1 / 3}\right)} \leq C_{2}\left(\left\|v_{k}\right\|_{L^{\infty}\left(B_{2 / 3}\right)}+\left[v_{k}\right]_{W^{s, 2}\left(B_{2 / 3}\right)}+\int_{\mathbb{R}^{n} \backslash B_{2 / 3}} \frac{\left|v_{k}(y)\right|}{|y|^{n+2 s}} d y\right),
$$

where $C_{2}=C_{2}(n, s, \lambda, \Theta, \varepsilon)>0$. For the first term of the right-hand side, in view of (61) and (60) we have

$$
\left\|v_{k}\right\|_{L^{\infty}\left(B_{2 / 3}\right)} \leq\left\|v_{k}\right\|_{L^{\infty}\left(B_{3 / 4}\right)} \leq\left\|v_{k}-w_{k}\right\|_{L^{\infty}\left(B_{3 / 4}\right)}+\left\|w_{k}\right\|_{L^{\infty}\left(B_{3 / 4}\right)} \leq \tau+2 .
$$

In order to estimate the tail term, we observe that by the same argument used in order to obtain (52), we have

$$
\left\|v_{k}-w_{k}\right\|_{L^{2}\left(B_{7 / 8}\right)} \leq C_{3} \delta
$$

where $C_{3}=C_{3}(n, s, \lambda, q)>0$. Together with the fact that $v_{k}=w_{k}$ in $\mathbb{R}^{n} \backslash B_{7 / 8}$, Lemma 2.2 and (60), we deduce

$$
\begin{aligned}
\int_{\mathbb{R}^{n} \backslash B_{2 / 3}} \frac{\left|v_{k}(y)\right|}{|y|^{n+2 s}} d y & \leq \int_{\mathbb{R}^{n} \backslash B_{2 / 3}} \frac{\left|w_{k}(y)\right|}{|y|^{n+2 s}} d y+\int_{B_{7 / 8} \backslash B_{2 / 3}} \frac{\left|v_{k}(y)-w_{k}(y)\right|}{|y|^{n+2 s}} d y \\
& \leq C_{4}\left(\left\|w_{k}\right\|_{L^{1}\left(B_{1}\right)}+\int_{\mathbb{R}^{n} \backslash B_{1}} \frac{\left|w_{k}(y)\right|}{|y|^{n+2 s}} d y+\int_{B_{7 / 8}}\left|v_{k}(y)-w_{k}(y)\right| d y\right) \\
& \leq C_{5}\left(\left\|w_{k}\right\|_{L^{\infty}\left(B_{1}\right)}+\int_{\mathbb{R}^{n} \backslash B_{1}} \frac{\left|w_{k}(y)\right|}{|y|^{n+2 s}} d y+\left\|v_{k}-w_{k}\right\|_{L^{2}\left(B_{7 / 8}\right)}\right) \leq C_{6},
\end{aligned}
$$

where $C_{6}=C_{6}(n, s, \lambda, q, \delta)>0$. Finally, for the Sobolev seminorm by Theorem 2.10 and the above estimates we have

$$
\left[v_{k}\right]_{W^{s, 2}\left(B_{2 / 3}\right)} \leq C_{7}\left(\left\|v_{k}\right\|_{L^{\infty}\left(B_{3 / 4}\right)}^{2}+\left\|v_{k}\right\|_{L^{\infty}\left(B_{3 / 4}\right)} \int_{\mathbb{R}^{n} \backslash B_{3 / 4}} \frac{\left|v_{k}(y)\right|}{|y|^{n+2 s}} d y\right) \leq C_{8},
$$

where $C_{7}$ and $C_{8}$ do not depend on $k$. By combining the above estimates with (62) and (63), we obtain that for any $x \in B_{1 / 3}$ we have

$$
\left|w_{k}(x)\right| \leq 2 \tau+C_{9}|x|^{\Theta-\varepsilon / 2},
$$

where again $C_{9}$ does not depend on $k$. Next, define

$$
w_{k+1}(x):=\frac{u\left(\rho^{k+1} x\right)-u(0)}{\rho^{k+1(\Theta-\varepsilon)}}=\frac{w_{k}(\rho x)}{\rho^{\Theta-\varepsilon}} .
$$


By choosing $\tau$ small enough such that $2 \tau<\rho^{\Theta}$, in view of (64), we obtain

$$
\left|w_{k+1}(x)\right| \leq 2 \tau \rho^{\varepsilon-\Theta}+C_{9} \rho^{\varepsilon-\Theta}|\rho x|^{\Theta-\varepsilon / 2} \leq\left(1+C_{9}|x|^{\Theta-\varepsilon / 2}\right) \rho^{\varepsilon / 2} \quad \forall x \in B_{\frac{1}{3 \rho}} .
$$

In particular, by choosing $\rho$ small enough such that $\rho \leq\left(1+C_{9}\right)^{-\frac{2}{\varepsilon}}$ and recalling that $\rho<1 / 3$, we arrive at $\left\|w_{k+1}\right\|_{L^{\infty}\left(B_{1}\right)} \leq 1$. By definition of $w_{k+1}$ this is equivalent to

$$
\sup _{x \in B_{\rho^{k+1}}}|u(x)-u(0)| \leq \rho^{(k+1)(\Theta-\varepsilon)}
$$

which proves the first estimate in (59) for $k+1$.

In order to prove the second estimate in (59) for $k+1$, we observe that (65) implies

$$
\begin{aligned}
\int_{B_{\frac{1}{3 \rho}} \backslash B_{1}} \frac{\left|w_{k+1}(y)\right|}{|y|^{n+2 s}} d y & \leq \rho^{\varepsilon / 2} \int_{B_{\frac{1}{3 \rho}} \backslash B_{1}} \frac{1+C_{9}|y|^{\Theta-\varepsilon / 2}}{|y|^{n+2 s}} d y \\
& \leq\left(1+C_{9}\right) \rho^{\varepsilon / 2} \int_{B_{\frac{1}{3 \rho}} \backslash B_{1}} \frac{d y}{|y|^{n+2 s+\varepsilon / 2-\Theta}} \leq C_{10} \rho^{\varepsilon / 2}
\end{aligned}
$$

where $C_{10}:=\left(1+C_{9}\right) \int_{\mathbb{R}^{n} \backslash B_{1}} \frac{d y}{|y|^{n+2 s+\varepsilon / 2-\Theta}}<\infty$ does not depend on $k$ and is finite because $2 s+\varepsilon / 2-\Theta \geq \frac{n}{q}+\varepsilon / 2>0$. Furthermore, by using a change of variables and the first bound in (60), we obtain

$$
\int_{B_{\frac{1}{\rho}} \backslash B_{\frac{1}{3 \rho}}} \frac{\left|w_{k+1}(y)\right|}{|y|^{n+2 s}} d y=\rho^{\varepsilon-\Theta+2 s} \int_{B_{1} \backslash B_{1 / 3}} \frac{\left|w_{k}(y)\right|}{|y|^{n+2 s}} d y \leq 2 \rho^{\varepsilon / 2} \int_{B_{1} \backslash B_{1 / 3}} \frac{d y}{|y|^{n+2 s}} \leq C_{11} \rho^{\varepsilon / 2},
$$

where $C_{11}:=3^{n+2 s} 2\left|B_{1}\right|<\infty$. Moreover, again by a change of variables and the second bound in (60), we deduce

$$
\int_{\mathbb{R}^{n} \backslash B_{\frac{1}{\rho}}} \frac{\left|w_{k+1}(y)\right|}{|y|^{n+2 s}} d y=\rho^{\varepsilon-\Theta+2 s} \int_{\mathbb{R}^{n} \backslash B_{1}} \frac{\left|w_{k}(y)\right|}{|y|^{n+2 s}} d y \leq M_{0} \rho^{\varepsilon / 2} .
$$

Note that in the last two estimates we also used that $\rho<1$ and that $\varepsilon-\Theta+2 s \geq \varepsilon / 2$. By combining the last three displays and choosing $\rho$ small enough such that

$$
\left(C_{10}+C_{11}+M_{0}\right) \rho^{\varepsilon / 2} \leq M_{0},
$$

we arrive at

$$
\int_{\mathbb{R}^{n} \backslash B_{1}} \frac{\left|w_{k+1}(y)\right|}{|y|^{n+2 s}} d y \leq\left(C_{10}+C_{11}+M_{0}\right) \rho^{\varepsilon / 2} \leq M_{0},
$$

which proves the second estimate in (59) for $k+1$. Therefore, for

$$
\rho<\min \left\{\frac{1}{3},\left(1+C_{9}\right)^{-\frac{2}{\varepsilon}}, M_{0}^{\frac{2}{\varepsilon}}\left(C_{10}+C_{11}+M_{0}\right)^{-\frac{2}{\varepsilon}}\right\}, \quad \tau<\frac{\rho^{\Theta}}{2}
$$

(59) is true for any $k \in \mathbb{N}_{0}$, which in particular also proves (58) under the assumptions (55) and (57), where $\delta$ is chosen as above.

Step 2: Regularity in a ball. Next, we show the desired higher Hölder regularity in the whole ball $B_{1 / 2}$. We fix some $0<\varepsilon<\Theta$ and take the corresponding small enough $\delta$ from step 1 . Fix $z \in B_{1 / 2}$, set $L:=2^{n+1}\left(1+\left|B_{1}\right|\right)$ and define

$$
u_{z}(x):=u\left(\frac{x}{2}+z\right) / L, \quad f_{z}(x):=\frac{2^{-2 s}}{L} f\left(\frac{x}{2}+z\right)
$$


and

$$
A_{z}(x, y):=A\left(\frac{x}{2}+z, \frac{y}{2}+z\right), \quad \widetilde{A}_{z}(x, y):=\widetilde{A}\left(\frac{x}{2}+z, \frac{y}{2}+z\right), \quad \Phi_{L}(t):=\frac{1}{L} \Phi(L t) .
$$

We note that $A_{z} \in \mathcal{L}_{0}(\lambda), \widetilde{A}_{z} \in \mathcal{L}_{1}\left(\lambda, B_{1}\right)$ and that $\Phi_{L}$ satisfies (4) and (5) with respect to $\lambda$. Moreover, $u_{z}$ is a local weak solution of $L_{A_{z}}^{\Phi_{L}} u_{z}=f_{z}$ in $B_{1}$ and by (55) we have

$$
\left\|A_{z}-\widetilde{A}_{z}\right\|_{L^{\infty}\left(\mathbb{R}^{n} \times \mathbb{R}^{n}\right)}=\|A-\widetilde{A}\|_{L^{\infty}\left(\mathbb{R}^{n} \times \mathbb{R}^{n}\right)} \leq \delta
$$

and

$$
\left\|f_{z}\right\|_{L^{q}\left(B_{1}\right)}=\frac{2^{n / q-2 s}}{L}\|f\|_{L^{q}\left(B_{1 / 2}(z)\right)} \leq\|f\|_{L^{q}\left(B_{1}\right)} \leq \delta .
$$

Additionally, by (57) we have

$$
\sup _{x \in B_{1}}\left|u_{z}(x)\right| \leq \sup _{x \in B_{1 / 2}(z)}|u(x)| \leq \sup _{x \in B_{1}}|u(x)| \leq 1
$$

and together with Lemma 2.2

$$
\begin{aligned}
\int_{\mathbb{R}^{n} \backslash B_{1}} \frac{\left|u_{z}(y)\right|}{|y|^{n+2 s}} d y & =\frac{2^{-2 s}}{L} \int_{\mathbb{R}^{n} \backslash B_{1 / 2}(z)} \frac{|u(y)|}{|y-z|^{n+2 s}} d y \\
& \leq \frac{2^{n}}{L} \int_{\mathbb{R}^{n} \backslash B_{1}} \frac{|u(y)|}{|y|^{n+2 s}} d y+\frac{2^{n}}{L}|| u \|_{L^{1}\left(B_{1}\right)} \\
& \leq \frac{2^{n}}{L} \int_{\mathbb{R}^{n} \backslash B_{1}} \frac{|u(y)|}{|y|^{n+2 s}} d y+\frac{2^{n}\left|B_{1}\right|}{L}\|u\|_{L^{\infty}\left(B_{1}\right)} \leq 1 .
\end{aligned}
$$

Therefore, we are in the position to apply step 1 to $u_{z}$, which yields

$$
\sup _{x \in B_{r}}\left|u_{z}(x)-u_{z}(0)\right| \leq C_{1} r^{\Theta-\varepsilon}, \quad 0<r<1 .
$$

By rewriting this estimate in terms of $u$, for any $z \in B_{1 / 2}$ we obtain

$$
\sup _{x \in B_{r}(z)}|u(x)-u(z)| \leq C_{1} L r^{\Theta-\varepsilon}, \quad 0<r<\frac{1}{2} .
$$

Now fix two points $x, y \in B_{1 / 2}$. Then applying (66) with $r=\frac{|x-y|}{2}<1 / 2$ and $z=(x+y) / 2$ yields

$$
\begin{aligned}
|u(x)-u(y)| \leq|u(x)-u(z)|+|u(y)-u(z)| & \leq 2 \sup _{\omega \in B_{r}(z)}|u(w)-u(z)| \\
& \leq 2 C_{1} L r^{\Theta-\varepsilon} \leq 2 C_{1} L|x-y|^{\Theta-\varepsilon},
\end{aligned}
$$

which proves the desired Hölder regularity of $u$.

In order to obtain the estimate (8) in our main results with its precise scaling, we now first prove Theorem 1.2 at scale 1 by using scaling and covering arguments. The general case will then follow by another scaling argument.

Theorem 4.3 Let $\lambda \geq 1$ and $f \in L^{q}\left(B_{1}\right)$ for some $q>\frac{n}{2 s}$. Consider a kernel coefficient $A \in \mathcal{L}_{0}(\lambda)$ and suppose that $\Phi$ satisfies (4) and (5) with respect to $\lambda$. Fix some $0<\alpha<$ $\min \left\{2 s-\frac{n}{q}, 1\right\}$. Then there exists some small enough $\delta=\delta(\alpha, n, s, \lambda, q)>0$, such that if 
for any $z \in B_{1}$, there exists some small enough radius $r_{z}>0$ and some $A_{z} \in \mathcal{L}^{1}\left(\lambda, B_{r_{z}}(z)\right)$ such that

$$
\left\|A-A_{z}\right\|_{L^{\infty}\left(B_{r_{z}}(z) \times B_{r_{z}}(z)\right)} \leq \delta
$$

then for any local weak solution $u \in W^{s, 2}\left(B_{1}\right) \cap L_{2 s}^{1}\left(\mathbb{R}^{n}\right)$ of the equation $L_{A}^{\Phi} u=f$ in $B_{1}$, we have $u \in C^{\alpha}\left(\bar{B}_{\sigma}\right)$ and

$$
[u]_{C^{\alpha}\left(B_{\sigma}\right)} \leq C\left(\|u\|_{L^{2}\left(B_{1}\right)}+\int_{\mathbb{R}^{n} \backslash B_{1}} \frac{|u(y)|}{|y|^{n+2 s}} d y+\|f\|_{L^{q}\left(B_{1}\right)}\right),
$$

where $C=C\left(n, s, \lambda, \alpha, \sigma, q,\left\{r_{z}\right\}_{z \in B_{1}}\right)>0$.

Proof Fix $\alpha \in(0, \Theta)$, where as before $\Theta=\min \left\{2 s-\frac{n}{q}, 1\right\}$, set $\varepsilon=\Theta-\alpha$ and let $\delta=\delta(\varepsilon, n, s, \lambda, q)>0$ be given by Proposition 4.2. We need to prove that $u \in C_{l o c}^{\Theta-\varepsilon}\left(\bar{B}_{\sigma}\right)$. Let $\delta=\delta(\varepsilon, n, s, \lambda, q)>0$ be the corresponding $\delta$ given by Proposition 4.2 and fix some $\sigma \in(0,1)$. Fix some $z \in \bar{B}_{\sigma}$. Then by assumption, there exists some small enough radius $r_{z} \in(0,1)$ with $B_{2 r_{z}}(z) \subset B_{1}$ and some kernel coefficient $A_{z} \in \mathcal{L}_{1}\left(\lambda, B_{r_{z}}(z)\right)$ such that

$$
\left\|A-A_{z}\right\|_{L^{\infty}\left(B_{r_{z}}(z) \times B_{r_{z}}(z)\right)} \leq \delta .
$$

Then the kernel coefficient

$$
\widetilde{A}(x, y):= \begin{cases}A_{z}(x, y) & \text { if }(x, y) \in B_{r_{z}}(z) \times B_{r_{z}}(z) \\ A(x, y) & \text { if }(x, y) \notin B_{r_{z}}(z) \times B_{r_{z}}(z)\end{cases}
$$

also belongs to $\mathcal{L}_{1}\left(\lambda, B_{r_{z}}(z)\right)$ and satisfies

$$
\|A-\widetilde{A}\|_{L^{\infty}\left(\mathbb{R}^{n} \times \mathbb{R}^{n}\right)} \leq \delta .
$$

In the case when $u \equiv 0$, the desired Hölder regularity trivially holds. Otherwise, set

$$
M_{z}:=\sup _{x \in B_{r_{z}}(z)}|u(x)|+r_{z}^{2 s} \int_{\mathbb{R}^{n} \backslash B_{r_{z}}(z)} \frac{|u(y)|}{|z-y|^{n+2 s}} d y+\frac{r_{z}^{2 s-n / q}}{\delta}\|f\|_{L^{q}\left(B_{r_{z}}(z)\right)}>0 .
$$

Consider the scaled functions $u_{1} \in W^{s, 2}\left(B_{1}\right) \cap L_{2 s}^{1}\left(\mathbb{R}^{n}\right)$ and $f_{1} \in L^{q}\left(B_{1}\right)$ given by

$$
u_{1}(x):=\frac{1}{M_{z}} u\left(r_{z} x+z\right), \quad f_{1}(x):=\frac{r^{2 s}}{M_{z}} f\left(r_{z} x+z\right)
$$

and also

$$
\begin{aligned}
A_{1}(x, y) & :=A\left(r_{z} x+z, r_{z} y+z\right), \quad \widetilde{A}_{1}(x, y):=\widetilde{A}\left(r_{z} x+z, r_{z} y+z\right), \\
\Phi_{1}(t) & :=\frac{1}{M_{z}} \Phi\left(M_{z} t\right) .
\end{aligned}
$$

We note that $u_{1}$ is a local weak solution of $L_{A_{1}}^{\Phi_{1}} u_{1}=f_{1}$ in $B_{1}$. Moreover, observe that $A_{1} \in \mathcal{L}_{0}(\lambda)$ and $\widetilde{A}_{1} \in \mathcal{L}_{1}\left(\lambda, B_{1}\right)$, while $\Phi_{1}$ satisfies (4) and (5) with respect to $\lambda$. Furthermore, by using changes of variables it is easy to verify that $u_{1}$ and $f_{1}$ satisfy

$$
\sup _{x \in B_{1}}\left|u_{1}(x)\right| \leq 1, \quad \int_{\mathbb{R}^{n} \backslash B_{1}} \frac{\left|u_{1}(y)\right|}{|y|^{n+2 s}} d y \leq 1, \quad\left\|f_{1}\right\|_{L^{q}\left(B_{1}\right)} \leq \delta,
$$

while (68) implies that

$$
\left\|A_{1}-\widetilde{A}_{1}\right\|_{L^{\infty}\left(\mathbb{R}^{n} \times \mathbb{R}^{n}\right)} \leq \delta
$$


Therefore, in view of (69) and (70) the assumptions (55) and (57) from Proposition 4.2 are verified with respect to $u_{1}, f_{1}, A_{1}$ and $\widetilde{A}_{1}$, so that by Proposition 4.2 we obtain

$$
\left[u_{1}\right]_{C^{\Theta-\varepsilon}\left(B_{1 / 2}\right)} \leq C_{1}(n, s, \lambda, q) .
$$

By rescaling and then using Theorem 2.11, we arrive at the estimate

$$
\begin{aligned}
{[u]_{C^{\Theta-\varepsilon}\left(B_{r_{z} / 2}(z)\right) \leq} } & \frac{C_{1}}{r_{z}^{\Theta-\varepsilon}}\left(\sup _{x \in B_{r_{z}}(z)}|u(x)|+r_{z}^{2 s} \int_{\mathbb{R}^{n} \backslash B_{r_{z}}(z)} \frac{|u(y)|}{|z-y|^{n+2 s}} d y\right. \\
& +\frac{r_{z}^{2 s-n / q}}{\delta}\|f\|_{\left.L^{q}\left(B_{r_{z}}(z)\right)\right)} \\
\leq & \frac{C_{2}}{r_{z}^{\Theta-\varepsilon}}\left(r_{z}^{-n / 2}|| u \|_{L^{2}\left(B_{2 r_{z}}(z)\right)}+r_{z}^{2 s} \int_{\mathbb{R}^{n} \backslash B_{r_{z}}(z)} \frac{|u(y)|}{|z-y|^{n+2 s}} d y\right. \\
& +r_{z}^{2 s-n / q}\|f\|_{\left.L^{q}\left(B_{2 r_{z}}(z)\right)\right)},
\end{aligned}
$$

where $C_{2}=C_{2}(n, s, \lambda, q, \Theta, \varepsilon)>0$. Since $\left\{B_{r_{z} / 4}(z)\right\}_{z \in \bar{B}_{\sigma}}$ is an open covering of $\bar{B}_{\sigma}$ and $\bar{B}_{\sigma}$ is compact, there exists a finite subcover $\left\{B_{r_{z_{i}} / 4}\left(z_{i}\right)\right\}_{i=1}^{N}$ of $\bar{B}_{\sigma}$ and hence of $B_{\sigma}$. Set

$$
r_{\min }:=\min _{i=1, \ldots, N} r_{z_{i}}>0
$$

Fix $x, y \in B_{\sigma}$ with $x \neq y$. Then $x \in B_{r_{z_{i}} / 4}\left(z_{i}\right)$ for some $i=1, \ldots, N$. If $|x-y|<r_{\min } / 4$, then in particular $y \in B_{z_{i}} / 2\left(z_{i}\right)$, so that by (71) and Lemma 2.2 we have

$$
\begin{aligned}
\frac{|u(x)-u(y)|}{|x-y|^{\Theta-\varepsilon}} & \leq[u]_{C^{\Theta-\varepsilon}\left(B_{r_{i} / 2}(z)\right)} \\
& \leq C_{3}\left(\|u\|_{L^{2}\left(B_{2 r_{z_{i}}}\left(z_{i}\right)\right)}+\int_{\mathbb{R}^{n} \backslash B_{r_{z_{i}}}\left(z_{i}\right)} \frac{|u(y)|}{\left|z_{i}-y\right|^{n+2 s}} d y+\|f\|_{L^{q}\left(B_{2 r_{z_{i}}}\left(z_{i}\right)\right)}\right) \\
& \leq C_{4}\left(\|u\|_{L^{2}\left(B_{1}\right)}+\int_{\mathbb{R}^{n} \backslash B_{1}} \frac{|u(y)|}{|y|^{n+2 s}} d y+\|f\|_{L^{q}\left(B_{1}\right)}\right),
\end{aligned}
$$

where $C_{3}$ and $C_{4}$ depend only on $n, s, \lambda, q, \Theta, \varepsilon$ and $r_{\min }$. If $|x-y| \geq r_{\min } / 4$, then in view of Theorem 2.11 and Lemma 2.2, we have

$$
\begin{aligned}
\frac{|u(x)-u(y)|}{|x-y|^{\Theta-\varepsilon}} & \leq 2\left(\frac{4}{r_{\min }}\right)^{\Theta-\varepsilon} \sup _{x \in B_{\sigma}}|u(x)| \\
& \leq C_{5}\left(\|u\|_{L^{2}\left(B_{1}\right)}+\int_{\mathbb{R}^{n} \backslash B_{\sigma}} \frac{|u(y)|}{|y|^{n+2 s}} d y+\|f\|_{L^{q}\left(B_{1}\right)}\right) \\
& \leq C_{6}\left(\|u\|_{L^{2}\left(B_{1}\right)}+\int_{\mathbb{R}^{n} \backslash B_{1}} \frac{|u(y)|}{|y|^{n+2 s}} d y+\|f\|_{L^{q}\left(B_{1}\right)}\right),
\end{aligned}
$$

where $C_{5}$ and $C_{6}$ depend only on $n, s, \lambda, q, \Theta, \varepsilon, \sigma$ and $r_{\min }$. Recalling that $\alpha=\Theta-\varepsilon$, combining the above estimates now proves the estimate (67) and in particular $u \in C^{\alpha}\left(\bar{B}_{\sigma}\right)$.

Proof of Theorem 1.2 Fix some $0<\alpha<\min \left\{2 s-\frac{n}{q}, 1\right\}$ and let $\delta=\delta(\alpha, n, s, \lambda, q)>0$ be given by Theorem 4.3. Fix $x_{0} \in \Omega$ and $R>0$ such that $B_{R}\left(x_{0}\right) \Subset \Omega$, so that by assumption for 
any $z^{\prime} \in B_{R}\left(x_{0}\right)$, there is some small enough radius $r_{z^{\prime}}>0$ and some $A_{z^{\prime}} \in \mathcal{L}^{1}\left(\lambda, B_{z_{z^{\prime}}}\left(z^{\prime}\right)\right)$ such that

$$
\left\|A-A_{z^{\prime}}\right\|_{L^{\infty}\left(B_{z_{z^{\prime}}}\left(z^{\prime}\right) \times B_{z_{z^{\prime}}}\left(z^{\prime}\right)\right)} \leq \delta .
$$

Consider the functions $u_{1} \in W^{s, 2}\left(B_{1}\right) \cap L_{2 s}^{1}\left(\mathbb{R}^{n}\right)$ and $f_{1} \in L^{q}\left(B_{1}\right)$ given by

$$
u_{1}(x):=u\left(R x+x_{0}\right), \quad f_{1}(x):=R^{2 s} f\left(R x+x_{0}\right)
$$

and also

$A_{1}(x, y):=A\left(R x+x_{0}, R y+x_{0}\right), \quad\left(A_{1}\right)_{z}(x, y):=A_{R z+x_{0}}\left(R x+x_{0}, R y+x_{0}\right), z \in B_{1}$, where $A_{R z+x_{0}}$ exists for any $z \in B_{1}$ since in this case we have $R z+x_{0} \in B_{R}\left(x_{0}\right)$. We note that for any $z \in B_{1}$ and $r_{z}:=r_{R z+x_{0}} / R>0$, we have $\left(A_{1}\right)_{z} \in \mathcal{L}^{1}\left(\lambda, B_{r_{z}}(z)\right)$ and

$$
\left\|A_{1}-\left(A_{1}\right)_{z}\right\|_{L^{\infty}\left(B_{r_{z}}(z) \times B_{r_{z}}(z)\right)} \leq \delta .
$$

In addition, $u_{1}$ is a local weak solution of $L_{A_{1}}^{\Phi} u_{1}=f_{1}$ in $B_{1}$. Therefore, by Theorem 4.3 along with some changes of variables, for any $\sigma \in(0,1)$ we obtain the estimate

$$
\begin{aligned}
R^{\alpha}[u]_{C^{\alpha}\left(B_{\sigma R}\left(x_{0}\right)\right)=} & {\left[u_{1}\right]_{C^{\alpha}\left(B_{\sigma}\right)} } \\
\leq & C\left(\left\|u_{1}\right\|_{L^{2}\left(B_{1}\right)}+\int_{\mathbb{R}^{n} \backslash B_{1}} \frac{\left|u_{1}(y)\right|}{|y|^{n+2 s}} d y+\left\|f_{1}\right\|_{L^{q}\left(B_{1}\right)}\right) \\
= & C\left(R^{-\frac{n}{2}}\|u\|_{L^{2}\left(B_{R}\left(x_{0}\right)\right)}+R^{2 s} \int_{\mathbb{R}^{n} \backslash B_{R}\left(x_{0}\right)} \frac{|u(y)|}{\left|x_{0}-y\right|^{n+2 s}} d y\right. \\
& \left.+R^{2 s-\frac{n}{q}}\|f\|_{L^{q}\left(B_{R}\left(x_{0}\right)\right)}\right),
\end{aligned}
$$

which proves the estimate (8). Furthermore, since $x_{0} \in \Omega$ is arbitrary, we in particular obtain that $u \in C_{l o c}^{\alpha}(\Omega)$.

Proof of Theorem 1.1 Fix some $0<\alpha<\min \left\{2 s-\frac{n}{q}, 1\right\}$ and let $\delta=\delta(\alpha, n, s, \lambda, q)>0$ be given by Theorem 1.2. Fix some $R>0$ and some $x_{0} \in \Omega$ with $B_{R}\left(x_{0}\right) \Subset \Omega$. Since $A$ satisfies (7) in $\Omega$ with respect to some $\varepsilon>0$, there exists some small enough $r_{\delta}>0$ such that

$$
\sup _{\substack{x, y \in B_{R}\left(x_{0}\right) \\|x-y| \leq \varepsilon}}|A(x+h, y+h)-A(x, y)| \leq \delta \quad \forall h \in B_{r_{\delta}} .
$$

Now fix some $z \in B_{R}\left(x_{0}\right)$ and some small enough radius $r_{z} \in(0,1)$ such that $r_{z} \leq$ $\min \left\{\varepsilon / 2, r_{\delta}\right\}$ and $B_{r_{z}}(z) \subset B_{R}\left(x_{0}\right)$. Then for all $x, y \in B_{r_{z}}(z)$ we have $z-y \in B_{r_{\delta}}$ and $z-x \in B_{r_{\delta}}$, so that (72) implies

$$
\sup _{x, y \in B_{r_{z}}(z)}|A(x-y+z, z)-A(x, y)| \leq \delta, \quad \sup _{x, y \in B_{r_{z}}(z)}|A(z, y-x+z)-A(x, y)| \leq \delta .
$$

Therefore, by additionally taking into account the symmetry of $A$, we see that the kernel coefficient defined by

$$
A_{z}(x, y):=\frac{1}{2}(A(x-y+z, z)+A(y-x+z, z))
$$

satisfies

$$
\left\|A-A_{z}\right\|_{L^{\infty}\left(B_{r_{z}}(z) \times B_{r_{z}}(z)\right)} \leq \delta
$$


and clearly belongs to the class $\mathcal{L}_{1}\left(\lambda, B_{r_{z}}(z)\right)$. Since $z \in B_{R}\left(x_{0}\right)$ is arbitrary, all assumptions from Theorem 1.2 are satisfied with $\Omega$ replaced by $B_{R}\left(x_{0}\right)$. Therefore, by Theorem 1.2 we see that the estimate (8) holds in any ball $B_{R}\left(x_{0}\right) \Subset \Omega$. In addition, since $x_{0} \in \Omega$ is arbitrary, we obtain that $u \in C_{l o c}^{\alpha}(\Omega)$.

Acknowledgements The author wants to thank the anonymous referee for careful reading and useful remarks that led to improvements of the paper.

Funding Open Access funding enabled and organized by Projekt DEAL.

Open Access This article is licensed under a Creative Commons Attribution 4.0 International License, which permits use, sharing, adaptation, distribution and reproduction in any medium or format, as long as you give appropriate credit to the original author(s) and the source, provide a link to the Creative Commons licence, and indicate if changes were made. The images or other third party material in this article are included in the article's Creative Commons licence, unless indicated otherwise in a credit line to the material. If material is not included in the article's Creative Commons licence and your intended use is not permitted by statutory regulation or exceeds the permitted use, you will need to obtain permission directly from the copyright holder. To view a copy of this licence, visit http://creativecommons.org/licenses/by/4.0/.

\section{References}

1. Brasco, L., Lindgren, E.: Higher Sobolev regularity for the fractional $p$-Laplace equation in the superquadratic case. Adv. Math. 304, 300-354 (2017)

2. Brasco, L., Lindgren, E., Schikkora, A.: Higher Hölder regularity for the fractional $p$-Laplacian in the superquadratic case. Adv. Math. 338, 782-846 (2018)

3. Brasco, L., Parini, E.: The second eigenvalue of the fractional p-Laplacian. Adv. Calc. Var. 9(5), 323-355 (2016)

4. Brasco, L., Santambrogio, F.: A sharp estimate à la Calderón-Zygmund for the $p$-Laplacian. Commun. Contemp. Math. 20(3), 521-573 (2018)

5. Caffarelli, L., Silvestre, L.: Regularity results for nonlocal equations by approximation. Arch. Ration. Mech. Anal. 200(1), 59-88 (2011)

6. Caffarelli, L., Stinga, P.: Fractional elliptic equations, Caccioppoli estimates and regularity. Ann. Inst. H. Poincaré Anal. Non Linéaire 33(3), 767-807 (2016)

7. Cozzi, M.: Interior regularity of solutions of non-local equations in Sobolev and Nikol'skii spaces. Ann. Mat. Pura Appl. (4) 196(2), 555-578 (2017)

8. Di Castro, A., Kuusi, T., Palatucci, G.: Local behavior of fractional p-minimizers. Ann. Inst. H. Poincaré Anal. Non Linéaire 33(5), 1279-1299 (2016)

9. Di Nezza, E., Palatucci, G., Valdinoci, E.: Hitchhiker's guide to the fractional Sobolev spaces. Bull. Sci. Math. 136(5), 521-573 (2012)

10. Dong, H., Kim, D.: On $L_{p}$-estimates for a class of non-local elliptic equations. J. Funct. Anal. 262(3), 1166-1199 (2012)

11. Fall, M.: Regularity results for nonlocal equations and applications, preprint, arXiv: 1806.09139 (2018)

12. Fall, M.: Regularity estimates for nonlocal Schrödinger equations. Discrete Contin. Dyn. Syst. 39(3), 1405-1456 (2019)

13. Giaquinta, M., Martinazzi, L.: An introduction to the regularity theory for elliptic systems, harmonic maps and minimal graphs, Lecture Notes. Scuola Normale Superiore di Pisa (New Series), 34, Second edition, (2012)

14. Grubb, G.: Fractional Laplacians on domains, a development of Hörmander's theory of $\mu$-transmission pseudodifferential operators. Adv. Math. 268, 478-528 (2015)

15. Kassmann, M.: A priori estimates for integro-differential operators with measurable kernels. Calc. Var. Part. Differ. Equ. 34(1), 1-21 (2009)

16. Korvenpää, J., Kuusi, T., Palatucci, G.: The obstacle problem for nonlinear integro-differential operators, Calc. Var. Partial Differential Equations 55(3), Art. 63, 29 (2016)

17. Kuusi, T., Mingione, G., Sire, Y.: Nonlocal equations with measure data. Commun. Math. Phys. 337(3), $1317-1368$ (2015)

18. Kuusi, T., Mingione, G., Sire, Y.: Nonlocal self-improving properties. Anal. PDE 8(1), 57-114 (2015) 
19. Leonori, T., Peral, I., Primo, A., Soria, F.: Basic estimates for solutions of a class of nonlocal elliptic and parabolic equations. Discrete Contin. Dyn. Syst. 35(12), 6031-6068 (2015)

20. Lindqvist, P.: Notes on the $p$-Laplace equation, Report. University of Jyväskylä, Department of Mathematics and Statistics, 102 (2006)

21. Mengesha, T., Schikorra, A., Yeepo, S.: Calderon-Zygmund type estimates for nonlocal PDE with Hölder continuous kernel, preprint, arXiv: 2001.11944 (2020)

22. Nowak, S.: $H^{s, p}$ regularity theory for a class of nonlocal elliptic equations. Nonlinear Anal. 195, 111730 (2020)

23. Ros-Oton, X., Serra, J.: Regularity theory for general stable operators. J. Differ. Equ. 260(12), 8675-8715 (2016)

24. Schikorra, A.: Nonlinear commutators for the fractional $p$-Laplacian and applications. Math. Ann. 366(12), 695-720 (2016)

25. Silvestre, L.: Hölder estimates for solutions of integro-differential equations like the fractional Laplace. Indiana Univ. Math. J. 55(3), 1155-1174 (2006)

Publisher's Note Springer Nature remains neutral with regard to jurisdictional claims in published maps and institutional affiliations. 